\title{
Processing of Intraoral Olfactory and Gustatory Signals in the Gustatory Cortex of Awake Rats
}

\author{
[DChad L. Samuelsen ${ }^{1}$ and Alfredo Fontanini ${ }^{2}$ \\ ${ }^{1}$ Department of Anatomical Sciences and Neurobiology, University of Louisville, Louisville, Kentucky 40292 and ${ }^{2}$ Department of Neurobiology and \\ Behavior and Program in Neuroscience, State University of New York at Stony Brook, Stony Brook, New York 11794
}

The integration of gustatory and olfactory information is essential to the perception of flavor. Human neuroimaging experiments have pointed to the gustatory cortex (GC) as one of the areas involved in mediating flavor perception. Although GC's involvement in encoding the chemical identity and hedonic value of taste stimuli is well studied, it is unknown how single GC neurons process olfactory stimuli emanating from the mouth. In this study, we relied on multielectrode recordings to investigate how single GC neurons respond to intraorally delivered tastants and tasteless odorants dissolved in water and whether/how these two modalities converge in the same neurons. We found that GC neurons could either be unimodal, responding exclusively to taste (taste-only) or odor (odor-only), or bimodal, responding to both gustatory and olfactory stimuli. Odor responses were confirmed to result from retronasal olfaction: monitoring respiration revealed that exhalation preceded odor-evoked activity and reversible inactivation of olfactory receptors in the nasal epithelium significantly reduced responses to intraoral odorants but not to tastants. Analysis of bimodal neurons revealed that they encode palatability significantly better than the unimodal taste-only group. Bimodal neurons exhibited similar responses to palatable tastants and odorants dissolved in water. This result suggested that odorized water could be palatable. This interpretation was further supported with a brief access task, where rats avoided consuming aversive taste stimuli and consumed the palatable tastants and dissolved odorants. These results demonstrate the convergence of the chemosensory components of flavor onto single GC neurons and provide evidence for the integration of flavor with palatability coding.

Key words: behavioral electrophysiology; gustatory cortex; hedonic; olfaction; palatability; taste

\section{Significance Statement}

Food perception and choice depend upon the concurrent processing of olfactory and gustatory signals from the mouth. The primary gustatory cortex has been proposed to integrate chemosensory stimuli; however, no study has examined the single-unit responses to intraoral odorant presentation. Here we found that neurons in gustatory cortex can respond either exclusively to tastants, exclusively to odorants, or to both (bimodal). Several differences exist between these groups' responses; notably, bimodal neurons code palatability significantly better than unimodal neurons. This group of neurons might represent a substrate for how odorants gain the quality of tastants.

\section{Introduction}

The ability to savor food and beverages depends on the interaction between gustatory and olfactory systems. Once in the mouth,

Received June 15, 2016; revised Nov. 8, 2016; accepted Nov. 11, 2016.

Author contributions: C.L.S. and A.F. designed research; C.L.S. performed research; C.L.S. and A.F. analyzed data; C.L.S. and A.F. wrote the paper.

This work was supported by National institute of Deafness and Other Communication Disorders Grant R01DC010389 to A.F. and Grant R03-DC014319 to C.L.S. We thank Dr. Roberto Vincis, Dr. Matthew Gardner, Dr. Haixin Liu, Martha Stone, Dr. Ahmad Jezzini, and Dr. Arianna Maffei for the very helpful comments and discussions; and Amy Cheung for help with histology.

The authors declare no competing financial interests.

Correspondence should be addressed to Dr. L. Chad Samuelsen, Department of Anatomical Sciences and Neurobiology, Medical Dental Research Building, Room 433, University of Louisville School of Medicine, Louisville, KY 40202. E-mail: chad.samuelsen@louisville.edu. nonvolatile chemicals from food dissolve in the saliva to activate taste receptors in the oral cavity, whereas volatile chemicals travel retronasally to activate olfactory receptors in the nasal epithelium. The concurrent activation of these anatomically separate chemosensory systems is crucial in giving food its flavor (Rozin, 1982; Verhagen and Engelen, 2006; Small, 2012). Although a vast body of work has explored the effects of odor and taste integration on chemosensory perception and preference, the convergence of these separate systems in the brain is a matter of debate. Traditional theories propose that the orbitofrontal cortex (OFC) is the first area capable of responding to both olfactory and gus- 
tatory stimuli (Rolls and Baylis, 1994; de Araujo et al., 2003; Rolls, 2005). However, recent evidence has challenged this view (Small et al., 2004, 2008; Veldhuizen et al., 2010; Maier et al., 2012, 2015), suggesting that both the primary olfactory and gustatory cortices themselves participate in the complex processing of multimodal chemosensory information (Small and Green, 2012).

Experiments examining concurrent processing of gustatory and olfactory stimuli have consistently found that gustatory cortex (GC) responds to both chemosensory stimuli. Human neuroimaging studies have shown that overlapping areas of GC respond to individual presentations of odorants (Savic et al., 2000; Cerf-Ducastel and Murphy, 2001; Poellinger et al., 2001) or tastants (Kobayakawa et al., 1999; Small et al., 1999, 2003; CerfDucastel et al., 2001; O’Doherty et al., 2001). Additionally, gustatory and olfactory sensory deficits have been observed in patients with GC lesions (Mak et al., 2005). However, no experiment has examined how intraoral gustatory and olfactory signals converge onto GC single units in behaving animals. The first step to understand the neural basis for this convergence in GC is to investigate how GC neurons represent individual gustatory and olfactory stimuli.

In the present study, all chemosensory stimuli were delivered directly into the oral cavity, via intraoral cannulae, to ensure that the odorants were detected by retronasal olfaction. Neurons in GC were divided into groups depending upon their responses to chemosensory stimuli. Unimodal neurons responded either exclusively to tastants (taste-only) or to odorants (odor-only), whereas bimodal neurons responded to both gustatory and olfactory stimuli. Reversible disruption of the olfactory epithelium confirmed that responses to intraoral delivery of odorants were the result of retronasal olfaction. Analysis of bimodal neurons revealed that they encoded palatable stimuli and predicted that odorized water could be palatable in our experimental conditions. This prediction was confirmed by a brief access behavioral task.

Together, these results provide novel evidence for GC single neuron processing of the chemosensory components responsible for the percept of flavor. Our results suggest that the coding of multimodal chemosensory information related to chemical identity and palatability is an integrative process.

\section{Materials and Methods}

Experimental subjects. All procedures were performed in accordance with university, state, and federal regulations regarding research animals and were approved by the Institutional Animal Care and Use Committee at Stony Brook University. Female Long-Evans rats ( $250-350 \mathrm{~g})$ were maintained on a $12 \mathrm{~h}$ light/dark cycle with ad libitum food and water unless otherwise noted.

Surgery. Rats were anesthetized with an intraperitoneal injection of a ketamine/xylazine/acepromazine mixture (KXA; 100, 5.2, and $1 \mathrm{mg} / \mathrm{kg}$ ). Surgical levels of anesthesia were maintained with supplemental doses ( $30 \%$ of the induction dose) when required. Once secured in a stereotaxic device, the animal's head was shaved, the scalp sterilized with an iodine mixture and excised to reveal the skull. Holes were drilled for the placement of anchoring screws and electrode bundles. Sixteen $25 \mu \mathrm{m}$ formvar-coated nichrome wire microdrivable electrode bundles were bilaterally implanted $0.5 \mathrm{~mm}$ dorsal to GC [anteroposterior $1.4 \mathrm{~mm}$, mediolateral $\pm 5 \mathrm{~mm}$ from bregma, dorsoventral $4 \mathrm{~mm}$ from dura]. To monitor respiration transients with an intranasal thermocouple, a subset of animals had a small hole made into the dorsal nasal recess (from frontal-nasal fissure: anteroposterior $0 \mathrm{~mm}$, mediolateral $0.9 \mathrm{~mm}$ ) (Wesson, 2013; Cazakoff et al., 2014) and a $17 \mathrm{~g}$ stainless steel guide cannulae with $21 \mathrm{~g}$ stainless-steel wire stylet was lowered $\sim 2 \mathrm{~mm}$ and fixed. All implants were cemented to the skull with dental acrylic. A head restraint bolt was cemented to the rear of the dental acrylic head cap and intraoral cannulae (IOCs) were bilaterally inserted to allow for the delivery of taste and odor stimuli. Rats were allowed to recover for 7-10 d before beginning behavioral training. Electrode placements were histologically verified using standard procedures (see Fig. 1A).

Recording procedure. Following recovery from implantation surgery, rats were placed on a water restriction regime ( $1 \mathrm{~h}$ access to water per day) and trained to sit calmly in restraint while receiving intraoral deliveries of tastants, odorants, or water. Stimuli were delivered via manifolds of polyimide tubes placed in the IOCs. Tastants consisted of sucrose $(0.1 \mathrm{M})$, $\mathrm{NaCl}(0.1 \mathrm{M})$, citric acid $(0.2 \mathrm{M})$, and quinine $(0.001 \mathrm{M})$. Odorants consisted of isoamyl acetate dissolved in water $(0.01 \%)$ and benzaldehyde in water $(0.01 \%)$. These two stimuli were chosen for their lack of gustatory component at the concentrations used here (Slotnick et al., 1997; Aimé et al., 2007; Julliard et al., 2007; Gautam and Verhagen, 2010, 2012; Tong et al., 2011; Rebello et al., 2015). Each trial began with a variable intertrial interval $(30 \pm 5 \mathrm{~s})$ at the end of which an $\sim 40 \mu \mathrm{l}$ aliquot of tastant, odorant, or water was delivered pseudo-randomly (pulse duration: $\sim 60$ $\mathrm{ms}$ ). An $\sim 50 \mu \mathrm{l}$ water rinse followed $5 \mathrm{~s}$ after each stimulus delivery. Each stimulus was delivered for 12 trials. A total of 84 trials (i.e., 7 stimuli $\times 12$ trials) were performed per session. After each session, animals were allowed $30 \mathrm{~min}$ free access to water in their home cage.

Electrophysiological and behavioral data recordings. Signals were recorded using standard multielectrode techniques (Samuelsen et al., 2012, 2013; Jezzini et al., 2013; Gardner and Fontanini, 2014). Briefly, 32 single-unit channels were simultaneously amplified, bandpass filtered (300 $-8 \mathrm{kHz}$ for single units), fed to a multichannel acquisition processor (Plexon; RRID:SCR_003170), and digitally acquired. Single units were isolated using a template algorithm, cluster-cutting, and examination of interspike interval plots (Plexon; Offline Sorter; RRID:SCR_000012). Data analysis was performed using Offline Sorter (Plexon; RRID: SCR_000012), Neuroexplorer (Nex Technologies; RRID:SCR_001818), and custom written scripts in MATLAB (The MathWorks, RRID: SCR_001622).

Analysis of single units. For each neuron, single trial activity and normalized peristimulus time histograms (PSTHs) were computed for all stimulus presentations. Neural activity was related to stimulus presentation by aligning action potential timestamps either to the stimulus delivery in the IOC or to the beginning of exhalation (see below). All responses were normalized using the area under the receiver-operating characteristic (auROC) (Cohen et al., 2012; Jezzini et al., 2013; Gardner and Fontanini, 2014). This method normalizes the stimulus-related activity to the baseline on a $0-1$ scale, where 0.5 represents the median of equivalence of the baseline activity. A score $<0.5$ is an inhibitory response, and $>0.5$ is excitatory. auROC values are derived from the probability that values in a given bin are higher or lower than the baseline activity. A score of 1 indicates that all values in the tested bin are greater than baseline, whereas a score of 0 indicates that all values are less than baseline. Population responses were obtained by averaging the auROC of each neuron in the observed population. A bin size of $100 \mathrm{~ms}$ was used for all analyses, unless otherwise specified.

Taste and odor responsiveness. Neurons were defined as taste-selective when two criteria were satisfied: (1) activity significantly differed from baseline; and (2) there was a significantly different response between the four tastants. Significance changes from baseline were established using a Wilcoxon rank-sum comparison between baseline bin and evoked bins with correction for family-wise error (FWE; Sidak correction or two consecutive significant bins, $p<0.01$ ). Significant difference evoked by the four tastants was determined relying on two-way ANOVA with Sidak correction for FWE ([taste $\times$ time], main effect for taste, $p<0.05$ ) for $100 \mathrm{~ms}$ bins over a $2.5 \mathrm{~s}$ window. Neurons were defined as odorresponsive when two criteria were satisfied: (1) activity significantly differed from baseline; and (2) responses to odorant and water were significantly different. Significance changes from baseline were established using a Wilcoxon rank-sum comparison between baseline bin and evoked bins with correction for FWE (Sidak correction or two consecutive significant bins, $p<0.01$ ). Significant difference between odorant and water was determined using a two-way ANOVA with Sidak correction or two consecutive significant bins for FWE ([odor $\times$ time], main effect for odor, $p<0.05$ ). Neurons that were inhibited by chemosensory 
stimuli were low in number and hard to evaluate due to the low spike count; therefore, our analysis focused on neurons that were excited by at least one of the chemosensory stimuli. Neurons that responded selectively to tastants, but not odorants, were designated "taste-only." Neurons that responded significantly to odorants, but not tastants, were designated as "odor-only." Finally, neurons that exhibited significant responses to tastants and odorants were classified as "bimodal." The proportion of neurons that responded only to a single tastant (or odorant) was compared with the proportion of neurons that responded to multiple tastants (or odorants) using a $\chi^{2}$ test $(p<0.05)$. For each taste-selective population, the proportions of taste-selective neurons that responded to each taste stimulus were compared using a $\chi^{2}$ test with the Tukey's honest significance test to correct for FWE. Stimulus onset latency was determined by averaging the time of the first bin that was significantly different from baseline for all neurons responsive to a given stimulus. Odor-onset latency was determined by averaging the time of the first bin that was significantly different from water for neurons responsive to odor stimuli. Stimulus peak latency was determined by averaging, for all neurons responsive to a given stimulus, the bin time that had the largest auROC score over a $2.5 \mathrm{~s}$ poststimulus analysis window. The onset and peak response latencies of the auROC-normalized population activity were compared between odor-responsive (odor-only vs bimodal) or taste-selective (taste-only vs bimodal) populations using a Wilcoxon rank-sum test $(p<0.05)$. The time course of taste selectivity was calculated by determining the number of taste-selective neurons per $100 \mathrm{~ms}$ bin. When a bin showed a significant difference across tastants (one-way ANOVA, $p<0.05$ ), that neuron was counted as taste-selective for that bin. A neuron could be counted as taste-selective for multiple bins. The time course of taste selectivity between the two taste-selective populations was compared with a $\chi^{2}$ test (correction of two consecutive significant bins, $p<0.05$ ). The time course for the breadth of tuning of the taste-selective neurons was calculated as follows. For each of the four taste stimuli, significant change from baseline was calculated for each 100 $\mathrm{ms}$ bin (Wilcoxon rank-sum, $p<0.05$ ). In each bin, a neuron was classified a responding to a single taste when there was significant change from baseline to only a single taste stimulus. A neuron was classified as responding to multiple tastants when it showed significant difference from baseline for two, three, or all four taste stimuli. For each tasteselective group, the tuning differences over time were compared with a $\chi^{2}$ test (correction of two consecutive significant bins, $p<0.05$ ). Comparisons in auROC-normalized taste-evoked activity between the tasteselective groups (taste-only vs bimodal) were performed using a Wilcoxon rank-sum test (correction of two consecutive significant bins, $p<0.05$ ). A Wilcoxon sign-rank test with correction for FWE (Sidak correction, $p<0.05$ ) was used to compare the auROC-normalized activity in the bimodal population's taste-, odor-, and water-evoked activity averaged across the period of palatability processing $(0-2 \mathrm{~s}$, see Palatability index). A further bin-by-bin analysis ( $250 \mathrm{~ms}$ bins) of the auROC-normalized response to sucrose, $\mathrm{NaCl}$, or odorants was compared with the activity evoked by water alone using a Wilcoxon sign-rank test (correction of two consecutive significant bins, $p<0.05$ ).

Palatability index. As measure of the palatability-related activity, we computed a palatability index (Fontanini et al., 2009; Piette et al., 2012; Sadacca et al., 2012; Jezzini et al., 2013; Li et al., 2013; Liu and Fontanini, 2015). This method considers the differences in evoked activity between taste stimuli with similar (sucrose $/ \mathrm{NaCl}$, citric acid/quinine) and opposite palatability (sucrose/quinine, $\mathrm{NaCl}$ /quinine, sucrose/citric acid, $\mathrm{NaCl} /$ citric acid). The auROC normalization method (see above) was used to estimate the differences between taste pairs. For each neuron, the absolute difference of the normalized taste-evoked activity between two pairs was calculated in $250 \mathrm{~ms}$ bins for every taste pair. The palatability index is defined as follows:

$$
P I=\left\langle D>_{\text {opposite }}-\left\langle D>_{\text {same }}\right.\right.
$$

where $\left\langle D>_{\text {opposite }}=\frac{1}{4}\left(a u R O C_{-} D_{S Q}+a u R O C_{-} D_{S C}+a u R O C_{-} D_{N Q}+\right.\right.$ auROC_D $\left.D_{N C}\right)$, and $\langle D\rangle_{\text {same }}=\frac{1}{2}\left(\right.$ auROC_D $D_{S N}+$ auROC_D $\left.D_{C Q}\right)$, subscripts represent taste pairs (e.g., $\mathrm{SN}$ indicates the taste pair of $\mathrm{S}$ and N). Significant change from baseline was established using the Wilcoxon rank-sum test $(p<$ 0.05 ). Comparison between taste-only and bimodal palatable indices was calculated with the Wilcoxon rank-sum test $(p<0.05)$. To investigate palatability-related activity of odor-evoked responses in the bimodal group, the palatability index was modified. To quantify the similarity of odorants to palatable/aversive pairs of taste stimuli, odor-evoked activity was compared with taste-evoked activity. The dissimilarity between responses to odorants and aversive tastants ("odor vs aversive") was computed by substituting isoamyl acetate and benzaldehyde for sucrose and $\mathrm{NaCl}$ in the palatability index formula. The dissimilarity between responses to odorants and palatable tastants ("odor vs palatable") was computed by substituting isoamyl acetate and benzaldehyde for citric acid and quinine in the palatability index formula. Significant change from baseline was established using the Wilcoxon rank-sum test $(p<$ 0.05). Difference between "odor vs aversive" and "odor vs palatable" palatability indices was ascertained with the Wilcoxon signed-rank test $(p<0.05)$.

Nasal inactivation with Triton $X-100$ (deciliation). To reversibly eliminate olfactory receptors, we followed the procedures outlined by FortisSantiago et al. (2010). Each rat was anesthetized with a mixture of KXA and placed on its side. A pliable $20 \mu$ l plastic pipette tip was inserted 15 $\mathrm{mm}$ into one nostril, and $\sim 50 \mu$ l solution of $0.125 \%$ Triton X-100 in saline was infused into the naris. The detergent (or saline control) solution was left in place for 5 min before being withdrawn back into the pipette tip. This procedure was repeated for the other nostril. After both nostrils underwent the procedure, the rat was placed in a prone position to allow drainage of any residual detergent. Rats were returned to their home cage to recover from anesthesia. Rats were allowed to recover overnight before undergoing recordings (see above). In a subset of sessions, single-unit responses to the chemosensory stimuli were recorded in the morning. Rats then underwent the nasal infusion, allowed at least $5 \mathrm{~h}$ to recover from anesthesia, and were then retested for chemosensory responses. Because of the indefinite period of time Triton X-100 application disrupts olfactory function (3-5 d or longer), within-neuron recordings before and after nasal inactivation were limited to one session per animal $(n=3)$. Neurons were defined as taste-selective or odorresponsive as above. Comparisons between the proportion of tasteselective and odor-responsive neurons in the normal condition versus saline application and the proportion of neurons after saline and after Triton X-100 were made using a $\chi^{2}$ test $(p<0.05)$.

Respiration monitoring and analysis. In a subset of animals $(n=4)$, recordings of respiration were performed with a Teflon-insulated thermocouple (0.13 mm OMEGA, part 5TC-TT-K-36-36). Only respiration transients that were stable throughout the entire recording session $(n=$ 13) were acquired for data analysis. Differential electric potentials were amplified (gain, 10,000 Hz; AM Systems, model 1700), bandpass filtered $(0.1-500 \mathrm{~Hz})$, and digitally acquired with a sampling rate of $1 \mathrm{kHz}(\mathrm{Mul}-$ tichannel Acquisition Processor; Plexon; RRID:SCR_003170). Acquisition of respiration traces was synchronized with video and neural activity recordings. Using custom MATLAB scripts, respiration data were normalized for peak amplitude and each respiratory peak time was detected. Each stimulus's average respiration latency was determined by averaging the timestamp of the first respiratory peak for each trial (the end of inhalation and the beginning of exhalation). The Kruskal-Wallis oneway ANOVA $(p<0.05)$ was used to determine whether stimulus type affected respiration latency. Frequency of respiration was calculated by averaging the number of respiratory peaks per second $2 \mathrm{~s}$ before and $5 \mathrm{~s}$ after each intraoral stimulus delivery. Respiration rate differences caused by stimulus delivery were determined with a Wilcoxon rank-sum test $(p<0.05)$. To determine the relationship between respiration and activity onset in the chemosensory responsive neuronal groups (odor-only, $n=5$; bimodal, $n=9$ ), onset latency of the average odor-evoked or taste-evoked response was compared with the corresponding respiration latency using the Wilcoxon rank-sum test $(p<0.05)$.

Brief access task. After completing the recording experiments, a subset of rats $(n=3)$ were tested for gustatory and olfactory preference with a 


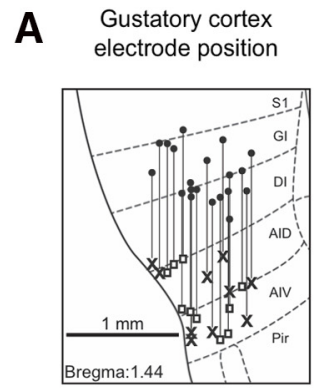

B Distribution of unimodal and bimodal neurons
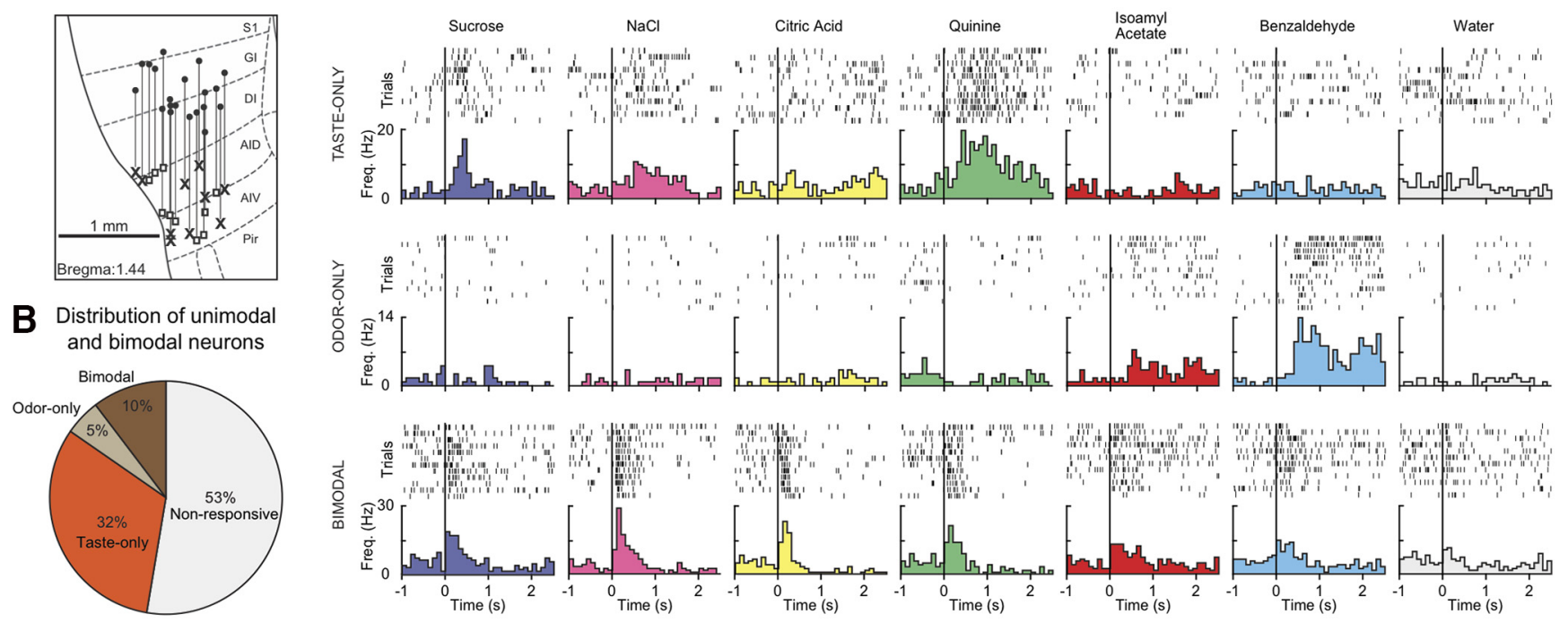

Figure 1. GC exhibits unimodal and bimodal responses to gustatory and olfactory stimuli. $A$, Schematic representation of the reconstructed path of the recording electrode positions in $G C$. Each line corresponds to the dorsoventral range of every movable electrode bundle. Filled circles represent the starting point of recordings. X indicates the final recording position in the right. Squares represent the left hemisphere. S1, Somatosensory cortex; GI, granular insular cortex; DI, disgranular insular cortex; AID, dorsal agranular insular cortex; AIV, ventral agranular insular cortex. B, Pie chart showing the distribution of chemoresponsive neurons in GC. The majority of neurons respond only to taste stimuli (102 of 319; taste-only, orange). The smallest group responds only to odor stimuli (16 of 319; odor-only, gray). Neurons that responded to both taste and odor stimuli were designated as bimodal (33 of 319; brown). C, Representative raster plots and PSTHs of a unimodal taste-only (top), unimodal odor-only (middle), and bimodal neuron (bottom). Each plot represents a single unit's response to the four tastants (sucrose [blue], NaCl [magenta], citric acid [yellow], and quinine [green]), two odorants (isoamyl acetate [red] and benzaldehyde [cyan]), and water (gray). Black vertical line indicates stimulus delivery (time $=0$ ).

brief access task (Smith et al., 1992; Sadacca et al., 2012). Rats were maintained on the same water restriction schedule as during the recording procedure. To habituate rats to the Davis MS-160 "brief access" Lickometer rig (DiLog Instruments), rats were allowed to drink water from a single tube continuously for $60 \mathrm{~min}$ during the first $2 \mathrm{~d}$. Next, rats were trained to lick during periodic brief access ( $15 \mathrm{~s}$ ) to one of seven water-filled lick tubes on a sliding tray for $60 \mathrm{~min}$ or 70 trials, whichever was completed first. The test sessions ( $n=5$ for each rat) consisted of each rat receiving periodic brief access to tastants $(0.1 \mathrm{M}$ sucrose, $0.1 \mathrm{M}$ $\mathrm{NaCl}, 0.2 \mathrm{M}$ citric acid, and $0.001 \mathrm{M}$ quinine), odorized-water $(0.01 \%$ isoamyl acetate and $0.01 \%$ benzaldehyde), and water. Presentations began with the raising of a steel shutter, exposing the lick spout. If in $60 \mathrm{~s} \mathrm{no}$ lick was registered, the shutter would close and a different tube was moved into place, these "miss" trials were removed from analysis (Sadacca et al., 2012). Once a lick was detected (via a low-current circuit), access was granted for $15 \mathrm{~s}$. After $15 \mathrm{~s}$, the shutter closed, another tube was moved into place and a $10 \mathrm{~s}$ intertrial interval began. Comparison of the mean number of licks between chemosensory stimuli was calculated using a one-way ANOVA and corrected for FWE with the Tukey's honest significance test $(p<0.05)$. Comparisons between the mean number of licks to water and the chemosensory stimuli were tested using a $t$ test $(p<$ $0.05)$. Stimuli with significantly lower mean numbers of licks than water were categorized as aversive and stimuli to which rats licked equally or greater than water were characterized as palatable.

Histology. At the end of experimental sessions, rats were terminally anesthetized with KXA and DC current ( $7 \mu \mathrm{A}$ for $7 \mathrm{~s}$ ) was applied to selected wires to mark electrode locations. Subjects were then transcardially perfused with saline followed by $10 \%$ formalin. The $80 \mu \mathrm{m}$ coronal sections were sliced with a cryostat. Using standard histological procedures, sections were stained with cresyl violet to track electrode locations (see Fig. 1A). One animal's electrode placement was unable to be reconstructed but was still included as the neural responses matched those of the other animals in the study.

\section{Results}

Ensembles of single neurons were recorded with chronic movable 16-wire electrode bundles bilaterally implanted in the GC of 11 rats. Figure $1 A$ shows the reconstructed position and the dorsalventral range of the recording electrodes. Once rats had recovered from surgery, they were habituated to restraint and trained to wait for the intraoral delivery of chemosensory stimuli. Each experimental session consisted of rats receiving pseudorandom intraoral deliveries of water, four gustatory stimuli $(0.1 \mathrm{M}$ sucrose, $0.1 \mathrm{M} \mathrm{NaCl}, 0.2 \mathrm{M}$ citric acid, $0.001 \mathrm{M}$ quinine), and two tasteless olfactory stimuli dissolved in water $(0.01 \%$ isoamyl acetate, 0.01\% benzaldehyde) (Slotnick et al., 1997; Aimé et al., 2007; Julliard et al., 2007; Gautam and Verhagen, 2010, 2012; Tong et al., 2011; Rebello et al., 2015). To investigate the effects of olfactory disruption on chemosensory responses in GC, subsets of sessions were performed after nasal inactivation, via olfactory receptor deciliation by the detergent Triton X-100 (Friedrich and Korsching, 1997). For the general experiment, 319 single units were recorded ( 44 sessions [7.25 units per session]). The nasal inactivation sessions consisted of a total of 98 single units recorded (saline condition: 7 sessions/59 neurons [8.4 units per session]; Triton X-100 condition: 7 sessions/39 neurons [5.6 units per session]).

\section{Unimodal and bimodal representations of gustatory and olfactory stimuli}

To determine the responsiveness of GC neurons to gustatory and olfactory stimuli, chemosensory-evoked activity was recorded after the intraoral delivery of individual tastants and odorants dissolved in water. Taste and odor responses were defined as follows (for details, see Materials and Methods). Taste-selective neurons must have: (1) exhibited a significant firing rate change from baseline and (2) shown significantly different responses to the four tastants. This criterion was chosen for consistency with prior work from our laboratory (Samuelsen et al., 2013; Gardner and Fontanini, 2014; Liu and Fontanini, 2015). Odor-responsive neurons must have: (1) exhibited a significant firing rate change from baseline and (2) shown 
A Effect of Triton X-100 inactivation

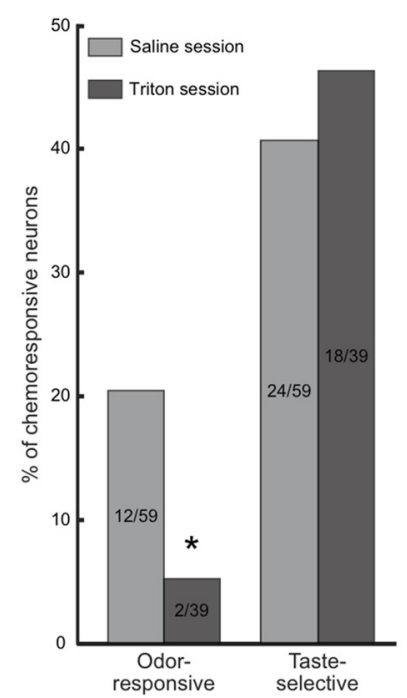

B
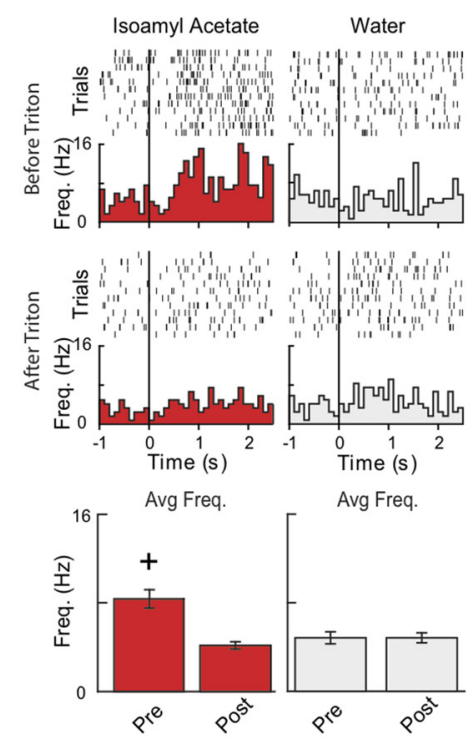

C
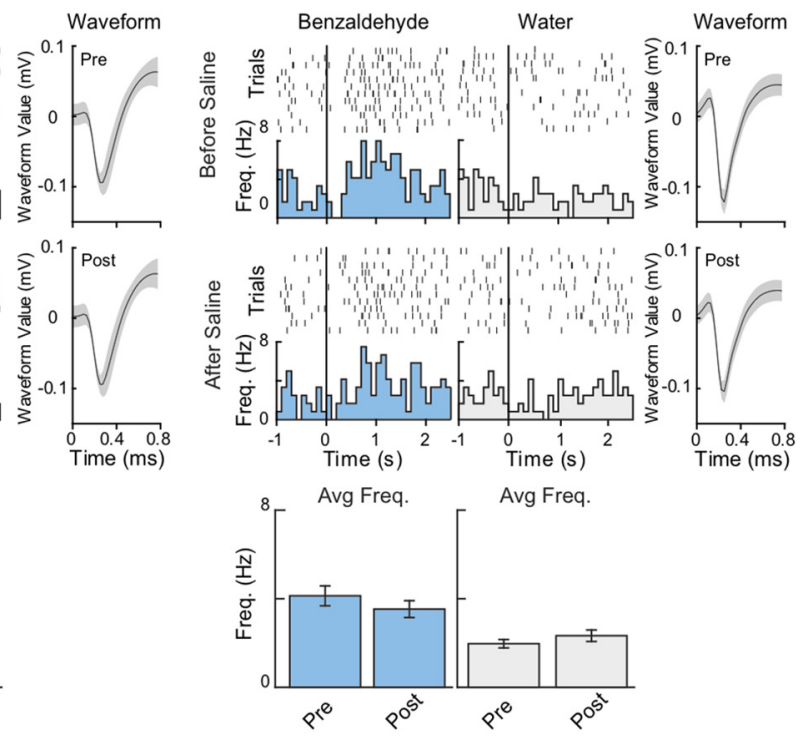

Figure 2. Olfactory receptor deciliation via nasal infusion of Triton X-100 disrupts responses to odorants in GC. A, Histogram represents the proportion of odor-responsive (left) and taste-selective (right) neurons recorded from control saline and Triton $X-100$ sessions. Relative to control saline infusion, the proportion of neurons that were responsive to intraoral odorant delivery was significantly reduced after Triton $\mathrm{X}-100$ treatment. ${ }^{*} p<0.05$. However, the proportion of taste-selective neurons was not different. Control infusion of saline did not alter the proportion of taste-selective or odor-responsive neurons relative to untreated animals. Left pair, Odor-responsive. Right pair, Taste-selective. Light gray represents saline session. Dark gray represents Triton X-100 session. $\boldsymbol{B}$, Raster plots, PSTHs, spike waveforms, and average poststimulus firing rate $(0-2.5 \mathrm{~s})$ of an odor-responsive neuron the morning before (top panels) and afternoon (middle panels) after Triton X-100 infusion. Triton X-100 treatment eliminated isoamyl acetate-evoked activity, significantly reducing the evoked firing rate (bottom left panel). ${ }^{+} p<0.01$. The infusion protocol did not alter baseline responses or response to water delivery (gray, middle panels). Black vertical line indicates stimulus delivery (time $=0$ ). C, Representative raster plots, PSTHs, spike waveforms, and average poststimulus firing rate of a benzaldehyde-responsive neuron the morning before (top panels) and afternoon after (middle panels) control saline infusion. The neuron remains responsive to benzaldehyde after saline (cyan, left panels). The infusion protocol did not alter either baseline responses or response to water delivery (gray, middle panels). Black vertical line indicates stimulus delivery (time $=0$ ).

significantly different responses from water. This double criterion for odor-responsive was purposefully stringent because the intraoral delivery of odorized-water solutions introduces the potential confound of somatosensory responsiveness to water. Neurons that met the above criteria for taste selectivity, but were not responsive to odorants, were classified as taste-only, the opposite criteria match were classified as odor-only, and those neurons that responded to both chemosensory stimuli were defined as bimodal. These three categories of chemoresponsive neurons exhibited different response distributions in GC (Fig. 1B). The majority of chemoresponsive neurons in GC showed unimodal responses to gustatory stimuli (32.0\%, 102 of 319) (Fig. 1C, representative example, top). The smallest group of chemoresponsive neurons exhibited unimodal olfactory responses $(5.0 \%, 16$ of 319$)$ (Fig. $1 C$, representative example, middle). The remaining group showed significant bimodal activity, responding to both gustatory and olfactory stimuli (10.4\%, 33 of 319) (Fig. $1 C$, representative example, bottom).

\section{Responses to intraorally delivered odorants}

The odorants used in this study were specifically chosen because they had previously been shown to be tasteless at these concentrations (Slotnick et al., 1997; Aimé et al., 2007; Julliard et al., 2007; Gautam and Verhagen, 2010, 2012; Tong et al., 2011; Rebello et al., 2015). However, GC responses to the intraoral delivery of odor stimuli may have resulted from residual taste receptor activation and not via retronasal olfaction. To determine whether odor responses in GC were the product of olfactory receptor activation, a subset of recording sessions were performed the day after the intranasal infusion of the mild detergent Triton X-100 $(0.125 \%$ in saline). This manipulation renders animals behavior- ally anosmic for short periods by eliminating the cilia of olfactory receptor neurons (Wachowiak and Cohen, 2001; Fortis-Santiago et al., 2010; Maier et al., 2012). Saline infusion did not significantly alter the proportion of neurons that responded to either taste stimuli (regular session: $42.3 \%, 135$ of 319 ; saline-control session: $40.6 \%, 24$ of $59, \chi^{2}, p>0.1$ ) or odor stimuli (regular session: $15.4 \%, 49$ of 319 ; saline session: $20.3 \%, 12$ of $59, \chi^{2}, p>$ 0.1 ) compared with the untreated population. Triton X-100 application significantly reduced the number of odor-responsive neurons in GC compared with saline application (saline session: 20.3\%, 12 of 59; Triton X-100 session: 5.1\%, 2 of $39, \chi^{2}, p<0.05$ (Fig. 2A, left). Importantly, Triton X-100 application did not affect the proportion of neurons that responded to taste stimuli (saline session: 40.6\%, 24 of 59; Triton X-100 session: 46.0\%, 18 of $\left.39, \chi^{2}, p>0.1\right)$ (Fig. 2A, right). Given the indefinite period of time Triton X-100 application disrupts olfactory function (3-5 d or longer), the ability to perform within-neuron recordings before and after inactivation was constrained. Even though restricted to a subset of sessions $(n=3)$, we recorded responses to chemosensory stimuli the morning before and the evening after nasal infusion in a representative group of neurons. Figure $2 B$ shows an example of an isoamyl acetate responsive neuron before (top left) and following treatment with Triton X-100 (middle left). Nasal infusion of Triton X-100 eliminated responsiveness to intraoral isoamyl acetate (bottom left) (before Triton: $8.37 \pm$ $0.83 \mathrm{~Hz}$; after Triton: $4.14 \pm 0.33 \mathrm{~Hz}$, Wilcoxon rank-sum, $p<$ 0.01 ). Control infusion of saline did not significantly reduce responsiveness to odorant. Figure $2 C$ shows a representative neuron that remained responsive to benzaldehyde after saline infusion (before saline: $4.13 \pm 0.45 \mathrm{~Hz}$; after saline: $3.53 \pm 0.38$ 
A

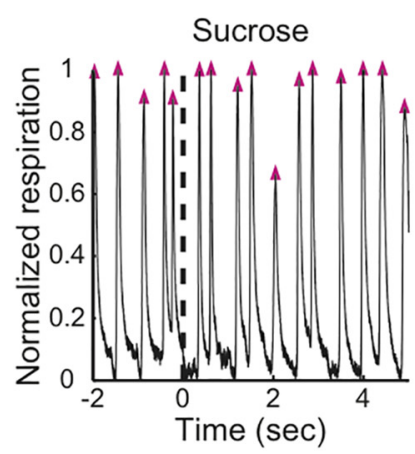

C Aligned to odor delivery

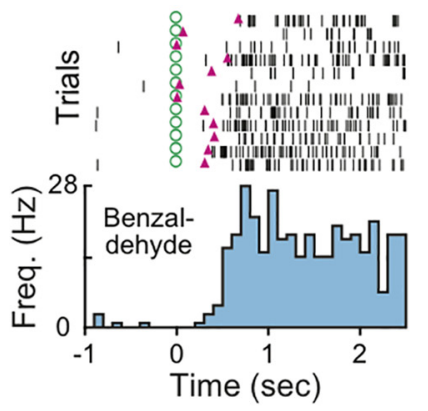

Representative respiration traces

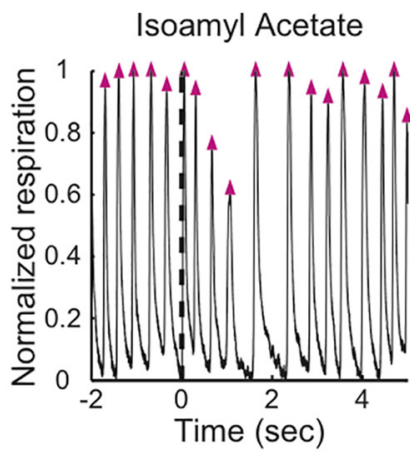

ve odor-only neuron Aligned to respiration

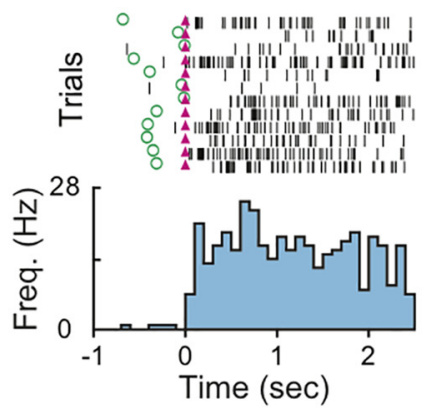

\section{B Odor-evoked response for odor-only neurons}

\section{Odor-evoked reponse for bimodal neurons}
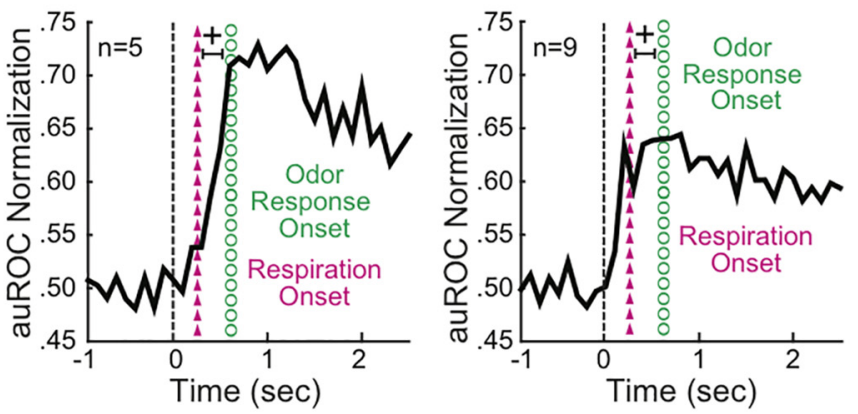

D

Representative bimodal neuron Aligned to odor delivery Aligned to respiration
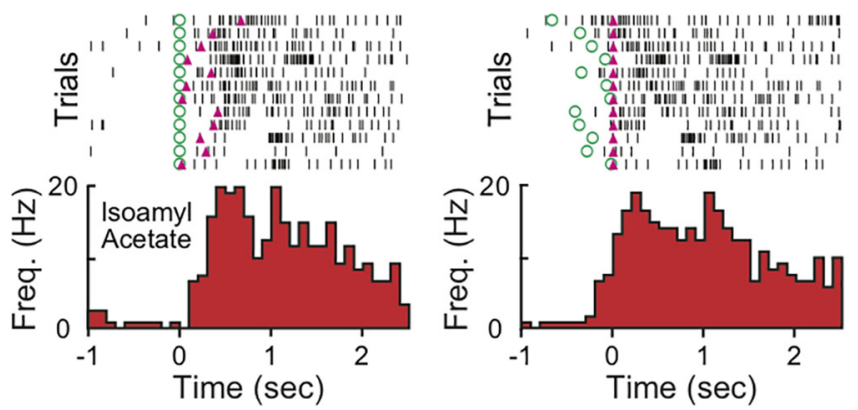

Figure 3. Response to intraoral odorants is linked to respiration. $\boldsymbol{A}$, Representative normalized respiration traces around the intraoral delivery (time $=0$, dashed line) of sucrose (left) and isoamyl acetate (right). Upward trajectory indicates inhalation; downward trajectory indicates exhalation. Each magenta triangle represents the time when inhalation ceases and exhalation begins. $\boldsymbol{B}$, Normalized population PSTHs (auROC) of odor-evoked responses recorded with respiration. Average odor-evoked activity onset (green dotted vertical line) occurred significantly after respiration onset (magenta triangle vertical line) in odor-only neurons ( $n=5$, left) and bimodal neurons $(n=9)$ (right). ${ }^{+} p<0.01$. C, Representative raster plots and PSTHs of an odor-only neuron aligned to intraoral odorant delivery and the first exhalation after stimulus delivery. Green dot represents stimulus delivery. Magenta triangle represents respiration onset. Benzaldehyde-evoked activity (cyan) aligned to intraoral delivery (left) and exhalation (right). Significant activity follows exhalation. $\boldsymbol{D}$, Representative raster plots and PSTHs of a bimodal neuron aligned to intraoral odorant delivery and the first exhalation after stimulus delivery. Isoamyl acetate-evoked activity (red) aligned to intraoral delivery (left) and exhalation (right). Significant activity occurs before exhalation.

$\mathrm{Hz}$, Wilcoxon rank-sum, $p>0.1$ ). Although some change in responsiveness may have been dependent upon time of day, the response to water delivery in both conditions did not differ between the morning and evening sessions (before Triton: $4.83 \pm 0.54 \mathrm{~Hz}$; after Triton: $4.183 \pm 0.45 \mathrm{~Hz}$, Wilcoxon ranksum, $p>0.1$; before saline: $1.97 \pm 0.19 \mathrm{~Hz}$; after saline: $2.33 \pm$ $0.26 \mathrm{~Hz}$, Wilcoxon rank-sum, $p>0.1$; Fig. $2 B, C$, bottom right). By disrupting olfactory function with infusion of Triton X-100, these results show that the odor responses in GC, which are elicited by intraoral odorant delivery, are dependent upon retronasal olfaction.

In a subset of sessions $(n=13)$, respiration was recorded during the intraoral delivery of chemosensory stimuli (Fig. $3 A$ ). Respiration rate did not significantly change from prestimulus delivery to poststimulus delivery for any of the stimuli (sucrose: Pre $2.12 \pm 0.13 \mathrm{~Hz}$, Post $2.30 \pm 0.16 \mathrm{~Hz}$; NaCl: Pre $2.15 \pm 0.14$ $\mathrm{Hz}$, Post $2.23 \pm 0.14 \mathrm{~Hz}$; citric acid: Pre $2.08 \pm 0.13 \mathrm{~Hz}$, Post $2.09 \pm 0.12 \mathrm{~Hz}$; quinine: Pre $2.11 \pm 0.13 \mathrm{~Hz}$, Post $2.32 \pm 0.14 \mathrm{~Hz}$; isoamyl acetate: Pre $2.20 \pm 0.15 \mathrm{~Hz}$, Post $2.29 \pm 0.16 \mathrm{~Hz}$; benzaldehyde: Pre $2.09 \pm 0.13 \mathrm{~Hz}$, Post $2.25 \pm 0.14 \mathrm{~Hz}$; water: Pre $2.04 \pm 0.13 \mathrm{~Hz}$, Post $2.09 \pm 0.12 \mathrm{~Hz}$, Wilcoxon rank-sum, $p>$ 0.1 ). Furthermore, the latency to the first inhalation after stimulus delivery did not differ among stimuli (sucrose: $238 \pm 29 \mathrm{~ms}$; $\mathrm{NaCl}: 279 \pm 31 \mathrm{~ms}$; citric acid: $246 \pm 14 \mathrm{~ms}$; quinine: $230 \pm 21$ ms; isoamyl acetate: $258 \pm 18 \mathrm{~ms}$; benzaldehyde: $286 \pm 35 \mathrm{~ms}$; water: $241 \pm 22 \mathrm{~ms}$, Kruskal-Wallis one-way ANOVA, $p>0.1$ ) with the average latency occurring $254 \pm 9 \mathrm{~ms}$ after stimulus delivery.

Next, we investigated the relationship between respiration and neural responses to intraoral odorant deliveries. The timing of the peak of the first inhalation after stimulus delivery was used to determine the onset of exhalation (i.e., the onset of retronasal smelling). The latency of odor-evoked activity was defined as the time in which the difference in firing rates evoked by odorized water and water was first significant. For unimodal odor-only neurons, in which we had consistent respiratory recordings $(n=5)$, we found that significant odor-evoked activity occurred only after the beginning of the first exhalation (Fig. 3B, left; representative example, Fig. $3 C$ ). Odor-evoked activity in unimodal odor-only neurons had a significantly slower latency compared with the first exhalation (first exhalation onset: $272 \pm 19 \mathrm{~ms}$; response latency: $663 \pm 127 \mathrm{~ms}$, Wilcoxon rank-sum, $p<0.01$ ). This delay indicated that the bulk of odor-specific neural activity was not triggered by the intraoral delivery of fluid, but rather by the exhalation of odorants. Bimodal neurons for which we had consistent respiratory record $(n=9)$ also exhibited a significantly slower onset of odor-evoked activity compared with the onset of exhalation (Fig. 3B, right; representative example, Fig. 3D) (first 
exhalation onset: $272 \pm 19 \mathrm{~ms}$; response latency: $670 \pm 108 \mathrm{~ms}$, Wilcoxon rank-sum, $p<0.01$ ). Furthermore, latencies of odorevoked activity did not differ between unimodal odor-only and bimodal neurons (unimodal latency: $663 \pm 127 \mathrm{~ms}$, bimodal latency: $670 \pm 108 \mathrm{~ms}$, Wilcoxon rank-sum, $p>0.1$ ). We found no relationship between odor-evoked activity and a specific phase of exhalation in GC neurons; however, this may be an effect of the small sample size. Future experiments will address in depth this important issue.

Visual inspection of the population response of bimodal neurons suggested that intraoral delivery of odorized water evokes a rapid increase in firing rates that may precede the onset of odorspecific activity (Fig. 3B, right). We defined the onset of this response as the first time bin in which stimulus-evoked activity differed from baseline. Bimodal neurons showed a significantly faster onset of stimulus-evoked activity compared with odorevoked activity (stimulus-evoked: $222 \pm 57$ ms; odor-evoked: $670 \pm 107 \mathrm{~ms}$, Wilcoxon sign-rank, $p<0.01)$. Delivery of odors dissolved in water resulted in an evoked response significantly faster in bimodal neurons than unimodal neurons (unimodal: $500 \pm 55$ ms, bimodal: $222 \pm 57 \mathrm{~ms}$, Wilcoxon sign-rank, $p<$ 0.01 ). The representative examples featured in Figure $3 C, D$ further confirmed that the activity evoked by intraoral delivery of an odorized solution followed the onset of exhalation in unimodal odor-only neurons, and preceded it in bimodal neurons. The fast activation of bimodal neurons by the intraoral delivery of fluid may reflect a response to oral stimulation.

Together, these data demonstrate that odor-evoked responses in GC to intraorally delivered odorants are the result of retronasal activation of olfactory receptors.

\section{Representation of gustatory stimuli in two populations of taste-selective GC neurons}

Next, we analyzed gustatory processing in the two groups of tasteselective neurons in GC: unimodal taste-only and bimodal. Figure $4 A$ shows the population responses and representative examples for each category. There was no significant difference between the two taste-selective groups in either taste-evoked response latency (sucrose: taste-only: $528 \pm 64 \mathrm{~ms}$; bimodal $603 \pm$ 98 ms, Wilcoxon rank-sum, $p>0.1$; NaCl: taste-only $462 \pm 69$ ms; bimodal $342 \pm 61 \mathrm{~ms}$, Wilcoxon rank-sum, $p>0.1$; citric acid: taste-only $459 \pm 62 \mathrm{~ms}$; bimodal $423 \pm 71 \mathrm{~ms}$, Wilcoxon rank-sum, $p>0.1$; quinine: taste-only $461 \pm 59 \mathrm{~ms}$; bimodal $648 \pm 160 \mathrm{~ms}$, Wilcoxon rank-sum, $p>0.1$ ) or taste-evoked peak activity (sucrose: taste-only $819 \pm 85 \mathrm{~ms}$; bimodal $979 \pm 136 \mathrm{~ms}$, Wilcoxon rank-sum, $p>0.1$; NaCl: taste-only $863 \pm 94 \mathrm{~ms}$; bimodal $777 \pm 125 \mathrm{~ms}$, Wilcoxon rank-sum, $p>0.1$; citric acid: taste-only $848 \pm 89 \mathrm{~ms}$; bimodal $841 \pm 156 \mathrm{~ms}$, Wilcoxon ranksum, $p>0.1$; quinine: taste-only $994 \pm 89$ ms; bimodal $952 \pm$ $186 \mathrm{~ms}$, Wilcoxon rank-sum, $p>0.1$ ). We did not find a relationship between $7-10 \mathrm{~Hz}$ rhythmicity and chemosensoryevoked responses in GC.

To identify potential group differences in the processing of gustatory information, we analyzed taste selectivity by calculating the proportion of neurons that responded to single or multiple tastants. A significantly greater proportion of unimodal tasteonly neurons responded to only single tastants (taste-only: $26 \%$, 26 of 102, vs bimodal: $9 \%, 3$ of $33 ; \chi^{2}, p<0.05$ ), whereas bimodal neurons responded with a greater proportion to multiple tastants (taste-only: $74 \%$, 76 of 102, vs bimodal: $91 \%, 30$ of 33; $\chi^{2}, p<$ $0.05)$. Regardless of the population difference in the proportion of sparse responding neurons, both groups responded significantly more to multiple tastants than to a single taste stimulus (taste-only single taste: $26 \%, 26$ of 102, vs taste-only multiple tastes: $74 \%, 76$ of 102, $\chi^{2}, p<0.01$; bimodal single taste: $9 \%, 3$ of 33 , vs bimodal multiple tastes: $91 \%, 30$ of $\left.33, \chi^{2}, p<0.01\right)$. We then calculated the prevalence of neurons that responded to each taste quality in both populations. Although neither group showed a significant difference in the proportion of neurons that responded across the four taste stimuli $\left(\chi^{2}, p>0.05\right)$, we did find a difference in responses between groups. Compared with tasteonly, bimodal neurons showed a significantly greater proportion of neurons responsive to sucrose (taste-only: 70.6\%, 72 of 102, vs bimodal: $87.9 \%, 29$ of $33, \chi^{2}, p<0.05$ ) and $\mathrm{NaCl}$ (taste-only: $56.7 \%$, 58 of 102, vs bimodal: 78.8\%, 26 of 33, $\chi^{2}, p<0.05$ ), whereas no difference was found for citric acid (taste-only: $62.7 \%$, 64 of 102, vs bimodal: $66.7 \%, 22$ of $33, \chi^{2}, p>0.1$ ) or quinine (taste-only: $64.7 \%, 66$ of 102 , vs bimodal $69.7 \%, 23$ of 33 , $\left.\chi^{2}, p>0.1\right)$. These analyses revealed that unimodal taste-only and bimodal neurons exhibit population differences in taste selectivity.

Next, we analyzed the temporal evolution of taste responses by calculating the time course of taste selectivity. The proportion of neurons that exhibited selective responses to taste stimuli was calculated for each $100 \mathrm{~ms}$ bin. Over a $2.5 \mathrm{~s}$ poststimulus timeframe, both populations responded selectively to tastants with a similar proportion and time course (Fig. 4B). The group of unimodal taste-only neurons exhibited a peak percentage of tasteselective neurons $(50.0 \%, 51$ of 102$)$ at $800 \mathrm{~ms}$ poststimulus onset. The group of bimodal neurons showed a similar peak percentage of taste-selective neurons $(48.5 \%, 16$ of 33$)$ shortly after, $1 \mathrm{~s}$ following stimulus delivery. The two groups did not significantly differ in their taste selectivity over time $\left(\chi^{2}, p>0.05\right)$. A similar temporal analysis was performed for taste tuning. For each $100 \mathrm{~ms}$ time bin, the proportion of neurons that responded to either a single taste or multiple tastants was calculated. The tuning time course for the unimodal taste-only group (Fig. 4C, left) showed that the response to multiple tastants peaked early ( $800 \mathrm{~ms} ; 40.2 \%, 41$ of 102 ) and then steadily declined, whereas the response to single tastants steadily rose and reached a plateau after $1.5 \mathrm{~s}$ (45.1\%, 46 of 102) (Fig. 4C, left, black bars, $\chi^{2}, p<$ 0.05 ). The same analysis on bimodal neurons (Fig. $4 C$, right) also revealed a peak in the response to multiple tastants at $800 \mathrm{~ms}$ $(54.6 \%, 18$ of 33$)$, but the peak response to single tastants occurred faster, at $600 \mathrm{~ms}(42.4 \%, 14$ of 33). Furthermore, at no point during the analyzed $2.5 \mathrm{~s}$ period did the proportions differ between dense and sparse tuning neurons (Fig. $4 C$, right $\chi^{2}, p>$ $0.05)$. Together, these findings indicate that, although the two taste-selective populations exhibit similar temporal profiles of taste selectivity, they have different tuning responses over time.

Next, we compared the activity evoked by each tastant for the two populations of neurons (Fig. 5A). The normalized population responses to sucrose or $\mathrm{NaCl}$ did not exhibit a significant difference between unimodal taste-only and bimodal groups at any time point during a $2.5 \mathrm{~s}$ analysis window (Fig. $5 A$, top). However, the activity evoked by citric acid and quinine showed significant differences between the unimodal taste-only and bimodal groups. Specifically, the bimodal group featured quinine and citric acid-evoked responses that were smaller and shorter compared with those observed in the unimodal taste-only group (Fig. 5A, bottom, black bars, Wilcoxon rank-sum, $p<0.05$ ). Visual inspection of these plots suggested that responses for bimodal neurons might group by palatability category with responses to palatable tastants being sustained longer than unpalatable ones. To test this possibility, we computed the palatability index (Fontanini et al., 2009; Piette et al., 2012; Sadacca et 


\section{A Population PSTH for taste-only neurons}

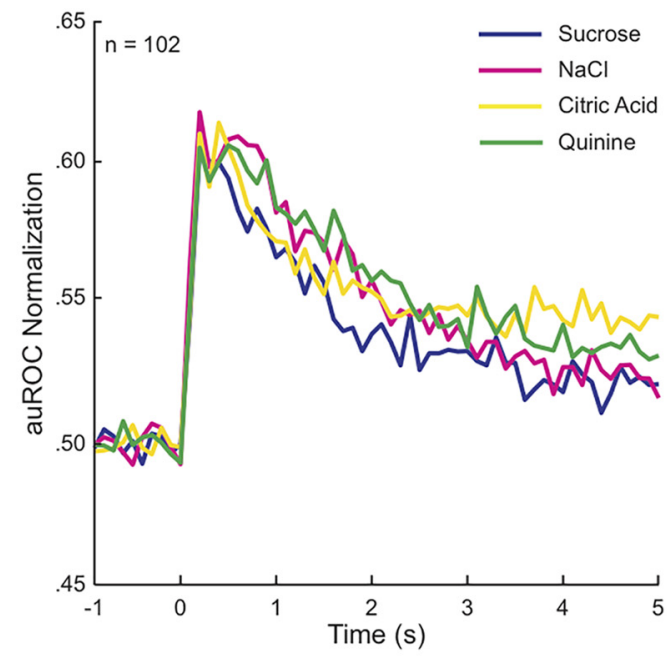

Population PSTH for bimodal neurons

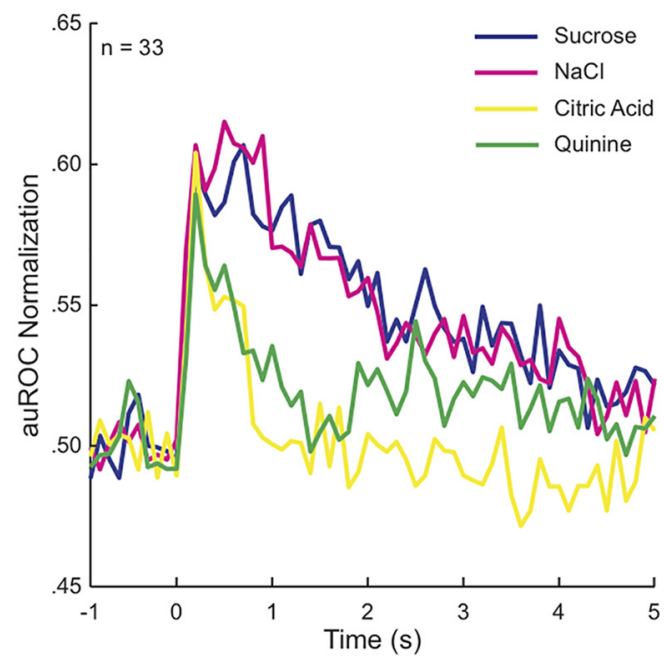

B

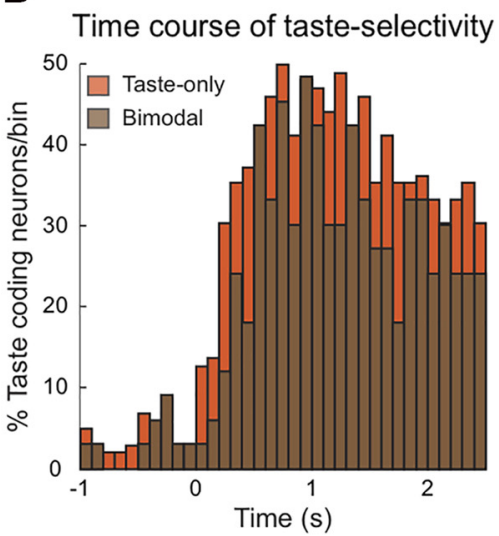

C

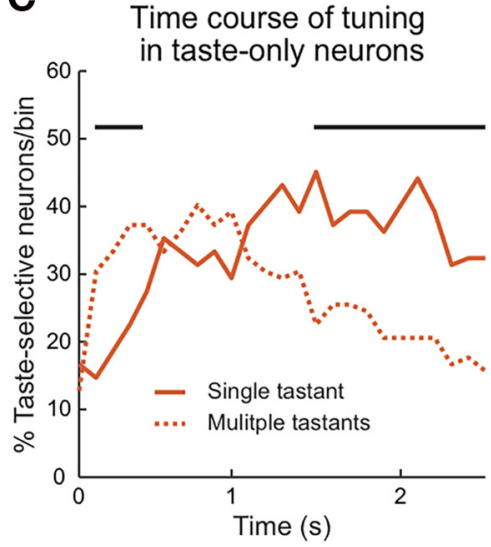

Representative taste-only neuron
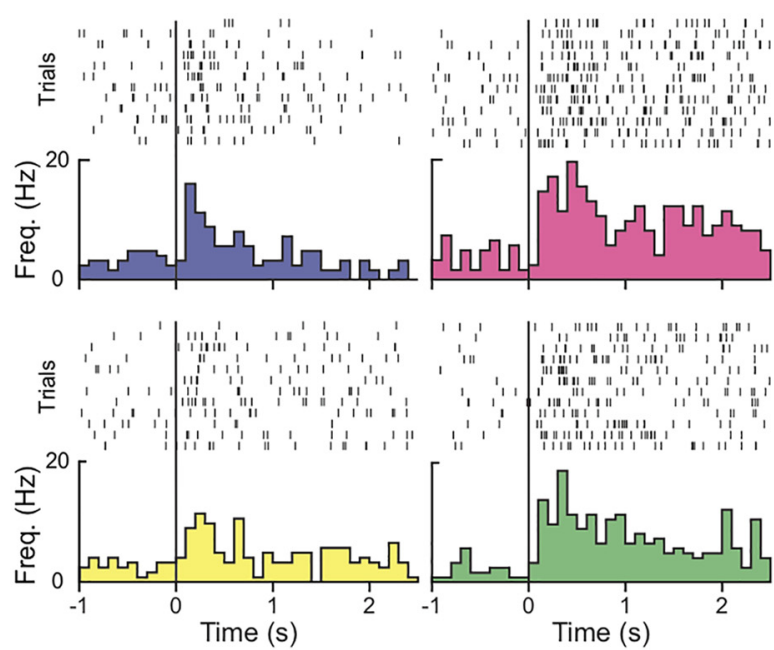

Representative bimodal neuron

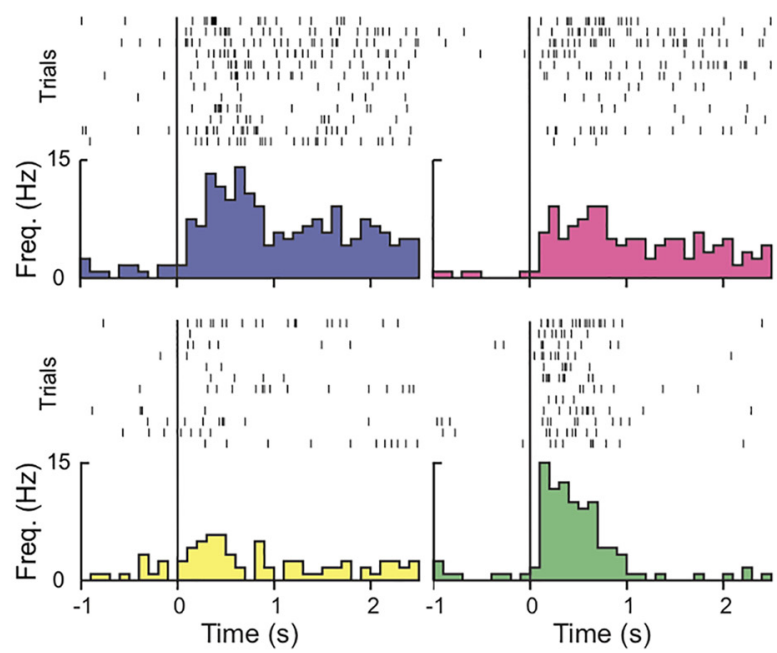

Time course of tuning in bimodal neurons

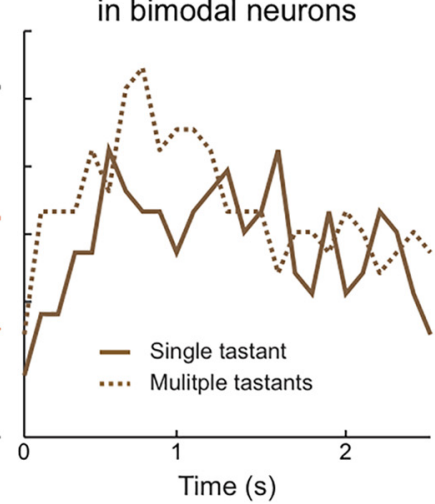

Figure 4. Unimodal and bimodal taste populations exhibit different taste-selective properties. $\boldsymbol{A}$, Normalized population PSTHs (auROC) and representative examples for taste-only ( $n=102$, top) and bimodal ( $n=33$, bottom) neurons in response to the intraoral delivery of taste stimuli (time $=0)$. Stimulus type: sucrose (blue), $\mathrm{NaCl}$ (magenta), citric acid (yellow), and quinine (green). $\boldsymbol{B}$, Proportion of taste-selective neurons during each successive $100 \mathrm{~ms}$ bin following intraoral taste delivery (time $=0$ ). The time course of taste selectivity did not differ between the taste-only (orange) and bimodal (brown) populations. C, Time course of tuning for taste-selective populations: taste-only (orange, left panel) and bimodal (brown, right panel), as measured by the proportion of taste-selective neurons responsive to single tastants (solid line) or multiple tastants (dashed line) within each $100 \mathrm{~ms}$ bin. Taste-only neurons are significantly more broadly tuned early and become significantly more sparsely tuned (responding to single tastants) over time (black bars, $p<0.05$ ). Bimodal neurons do not exhibit significant differences in tuning over time. Neurons responding selectively to one taste within a bin could respond to another or multiple tastants in a different bin. 


\section{A}

Taste-evoked activity in taste-only and bimodal neurons

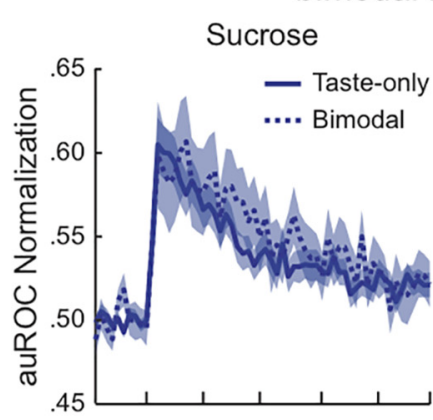

Citric Acid
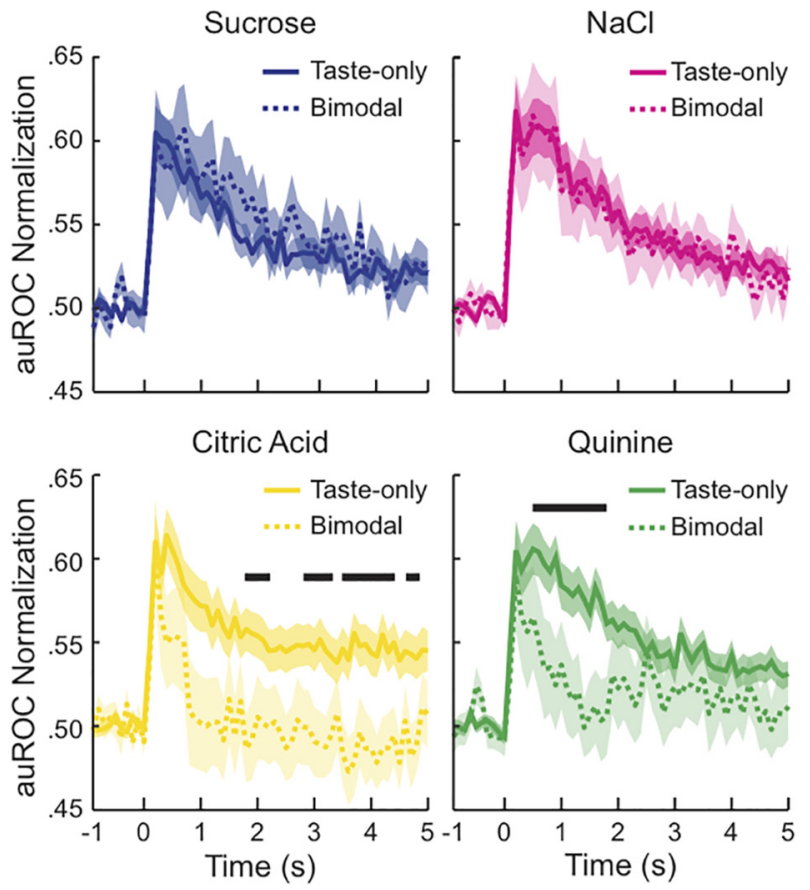

B

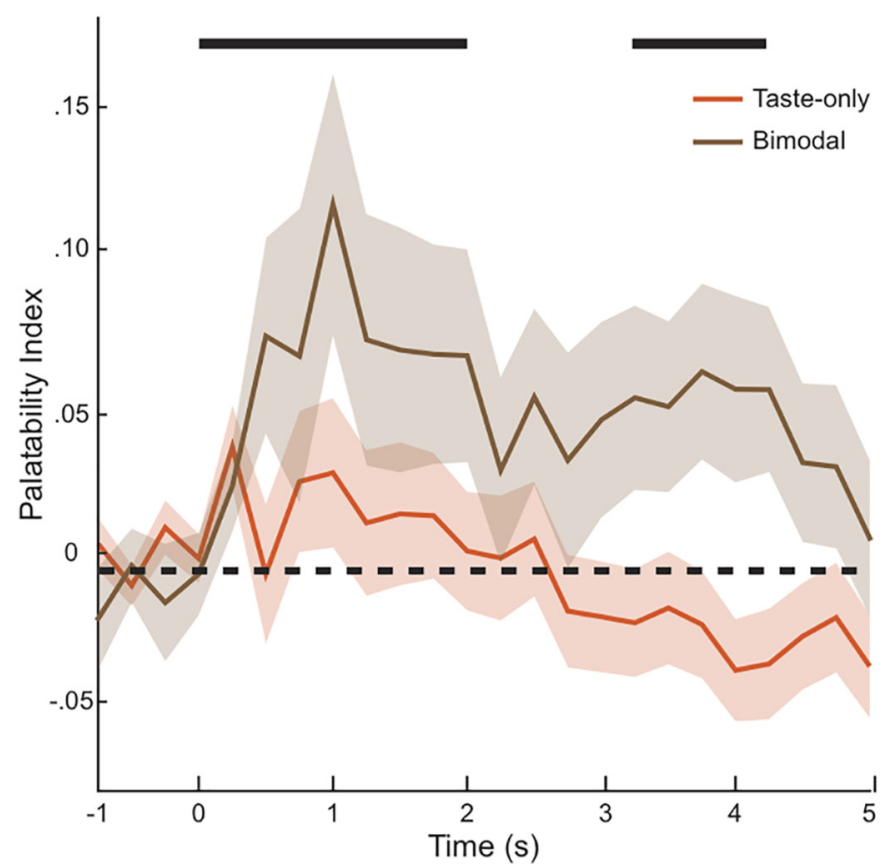

Figure 5. Bimodal neurons represent palatability. $\boldsymbol{A}$, Normalized population PSTHs (auROC) for taste-only ( $n=102$, solid lines) and bimodal ( $n=33$, dashed lines) neurons in response to intraoral delivery of either palatable tastants [sucrose (top left panel, blue) and $\mathrm{NaCl}$ (top right panel, magenta)] or aversive tastants [citric acid (bottom left panel, yellow) and quinine (bottom right panel, green)]. Taste-evoked activity does not differ between the taste-selective groups for palatable tastants (sucrose and NaCl) but is significantly different for aversive tastants (citric acid and quinine). Black bars represent bins with significant differences $(p<0.05)$ between unimodal taste-only and bimodal neurons. Shaded area represents $S E M$. $\boldsymbol{B}$, Comparison of the time course of palatability coding between taste-only $(n=103)$ and bimodal $(n=33)$ populations. Each solid line indicates the palatability index (see Materials and Methods) computed for taste-only (orange) and bimodal (brown) neurons. Black dashed line indicates baseline. Shaded area represents SEM. The bimodal group codes palatability significantly better than the taste-only population ( $p<0.01$ ). The bimodal population exhibits multiple time points (black bars, $p<0.05$ ) when palatability coding is significantly higher than baseline. At no time point did the taste-only population show significant difference from baseline.

al., 2012; Jezzini et al., 2013; Li et al., 2013; Liu and Fontanini, 2015) for neurons in the two groups. Briefly, this analysis compares the activity evoked by tastants with similar palatability (i.e., sucrose $/ \mathrm{NaCl}$ and citric acid/quinine) with activity evoked by hedonically dissimilar tastants (i.e., sucrose/citric acid and sucrose/quinine, etc.; for details, see Materials and Methods). Figure $5 B$ shows that, on average, the population of bimodal neurons significantly encodes palatability following intraoral stimulus delivery (brown line, black bars, Wilcoxon rank-sum, $p<0.05$ ), whereas on average the population of unimodal tasteonly does not represent palatability at any point during the $5 \mathrm{~s}$ analysis timeframe (orange line, Wilcoxon rank-sum, $p>0.05$ ). This difference in palatability coding between the two tasteselective groups was significant (Wilcoxon rank-sum, $p<0.01$ ).

Next, we determined the number of palatability coding neurons in the two taste-selective populations. A significantly greater proportion of bimodal neurons (16 of 33, 48\%) encoded palatability compared with unimodal taste-only neurons (23 of 102, $\left.23 \% ; \chi^{2}, p<0.01\right)$. Although not sufficient to drive a significant change in the population palatability index (Fig. $5 B$ ), unimodal taste-only palatability encoding neurons made up 15 of the 20 largest palatability scores. We next assessed whether the different palatability coding populations showed a response bias to palatable or aversive taste stimuli. Both bimodal and unimodal tasteonly palatability coding neurons showed a bias toward palatable tastants; however, the two populations did significantly differ. All of the bimodal neurons showed the greatest response to palatable tastants, whereas a significantly smaller proportion of unimodal taste-only neurons showed the greatest response to palatable tastants (bimodal: 16 of $16,100 \%$ strongest response to $\mathrm{S} / \mathrm{N}$; unimodal: 17 of $23,74 \%$ strongest response to S/N; $\chi^{2}, p<0.05$ ). These results indicate that bimodal neurons are particularly effective in coding palatability and are biased to palatable tastants but are not the sole source of palatability encoding neurons in GC.

\section{Palatability of odorized water solutions}

The results presented above show that bimodal neurons encode palatability and have a bias for palatable tastants. Because water is palatable to thirsty rats, odors dissolved in water may be palatable as well and evoke activity similar to that of sucrose and $\mathrm{NaCl}$ in bimodal neurons. To address this point, we compared responses to tastants and odorized water in bimodal neurons. Figure $6 \mathrm{~A}$ shows the population activity (and representative example) evoked by odorants (dotted traces) overlaid on the responses to taste stimuli and water for the bimodal population. Population responses to solutions of isoamyl acetate or benzaldehyde overlapped with responses to sucrose and $\mathrm{NaCl}$. Specifically, responses to odorants, like those to sucrose and $\mathrm{NaCl}$, appeared to last longer than responses to aversive tastants. The similarity suggests that the solutions of water and odorants used in this study could be perceived as palatable. Next, we performed analyses to compare the activity evoked by odorants, tastants, and water. Normalized activity of the bimodal population was averaged 


\section{A Population PSTH for bimodal neurons}

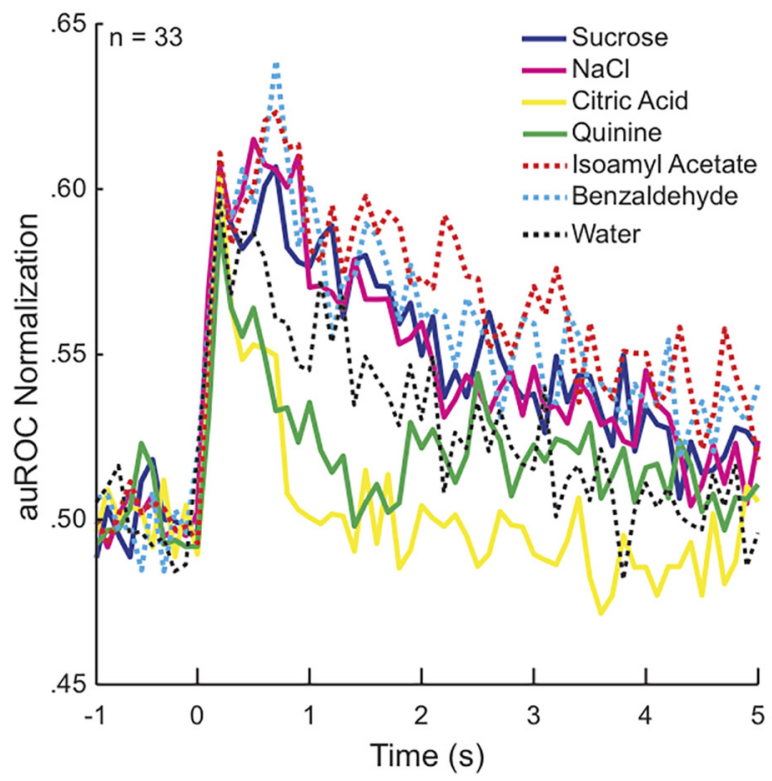

B

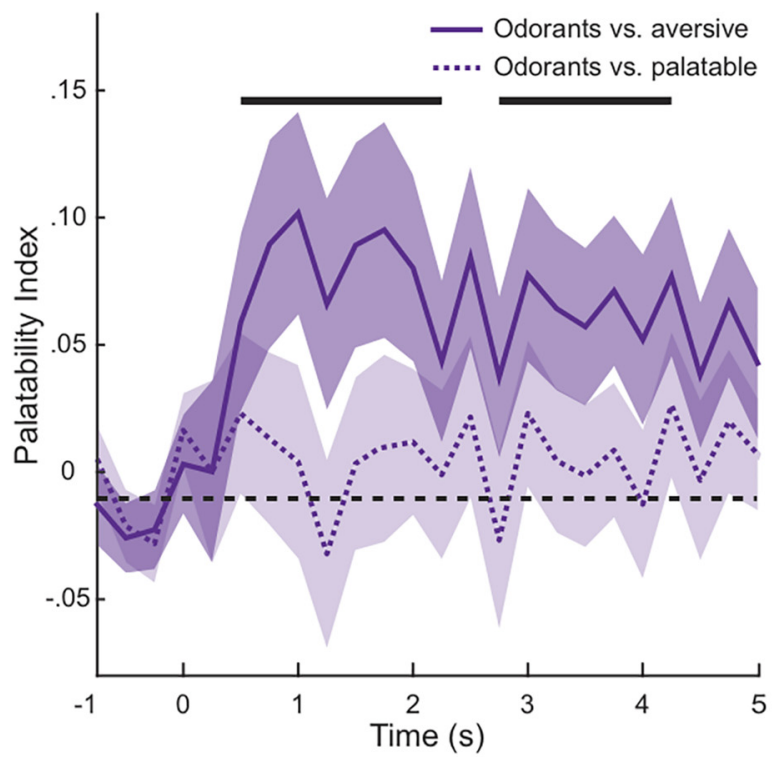

Representative bimodal neuron
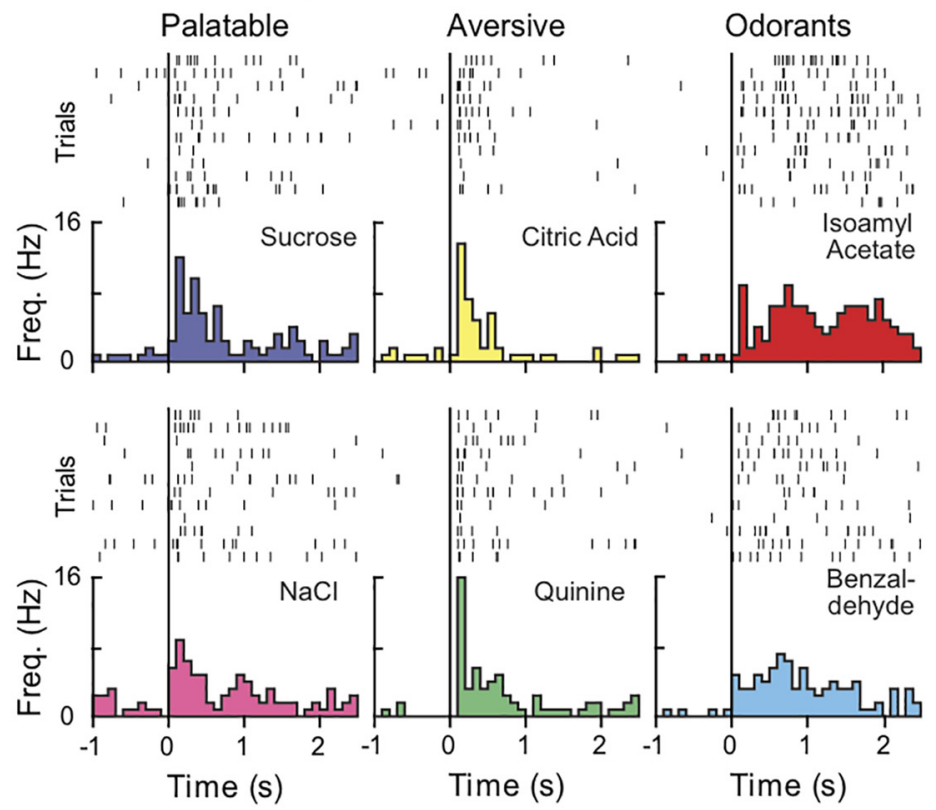

C

Brief access task

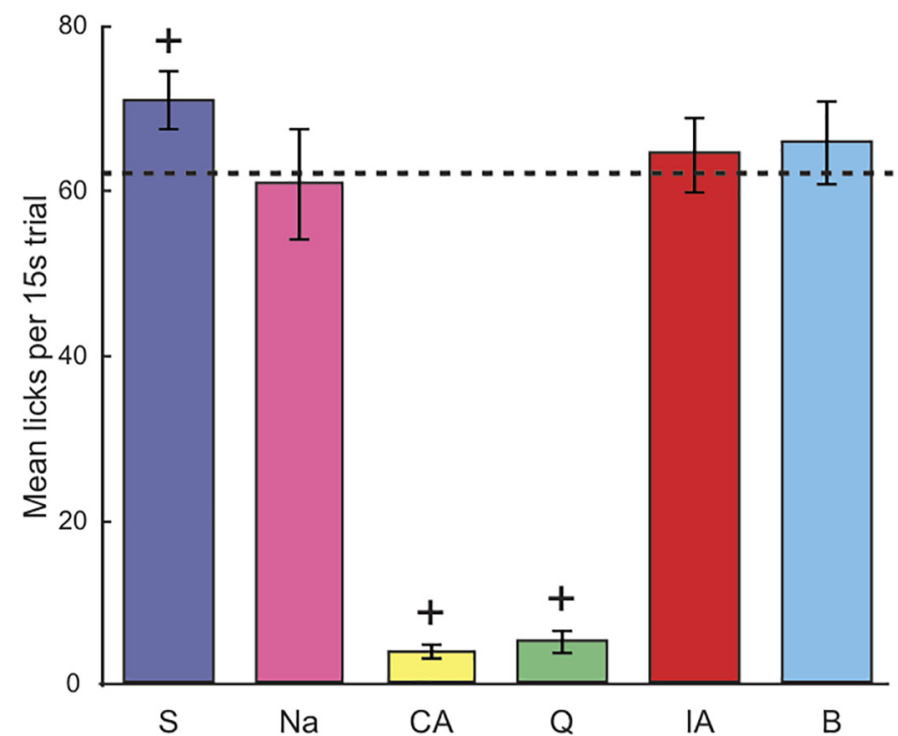

Figure 6. Delivery of odorants and palatable tastants evokes similar responses. $A$, Normalized population PSTHs for bimodal neurons (left) and representative example (right) featuring responses to the intraoral delivery of chemosensory stimuli (time $=0$ ). Stimulus type: sucrose (blue), $\mathrm{NaCl}$ (magenta), citric acid (yellow), quinine (green), isoamyl acetate (red), benzaldehyde (cyan), and water (black). $\boldsymbol{B}$, Comparison of the bimodal groups' $(n=33$ ) odor-evoked activity to palatable/aversive tastant-activity using a modified palatability index. Odorants were significantly different from aversive taste stimuli relative to baseline (purple solid line, black bars, $p<0.05$ ). No difference was observed between responses to odors and to palatable taste stimuli (purple dashed line). The modified palatability indices were significantly different from each other $(p<0.01)$. The black dashed line indicates baseline. Shaded area represents SEM. C, Histogram of the mean number of licks during a brief access task. As predicted by the neural activity of $\mathrm{GC}$ bimodal neurons, rats exhibited significantly fewer licks to the aversive tastants (citric acid and quinine) compared with water. ${ }^{+} p<0.01$. Rats did not exhibit a significant difference in the number of licks to $\mathrm{NaCl}$, isoamyl acetate or benzaldehyde but did lick significantly more to sucrose. ${ }^{+} p<0.01$. The black dashed line indicates average number of licks to water. Rats show similar consummatory behaviors to experienced odorants and palatable tastants while avoiding aversive taste stimuli.

across neurons for the period of palatability processing $(0-2 \mathrm{~s}$, Fig. $5 B$ ). Activity evoked by odorized water (isoamyl acetate and benzaldehyde) was not different from the activity evoked by sucrose (Wilcoxon sign-rank, $p>0.05$ ) or $\mathrm{NaCl}$ (Wilcoxon signrank, $p>0.05$ ) but was significantly different from citric acid (Wilcoxon sign-rank, $p<0.001$ ) and quinine (Wilcoxon sign- rank, $p>0.001)$. Interestingly, odor-evoked activity, just as sucrose (Wilcoxon sign-rank, $p<0.001$ ) and $\mathrm{NaCl}$ (Wilcoxon sign-rank, $p<0.001$ ), was significantly greater than the response to water alone (Wilcoxon sign-rank, $p<0.001$ ). A bin $\times$ bin comparison (250 ms bins) of the time course of palatable tastant-, odorant-, and water-evoked activity in the population of bimodal 
neurons found that sucrose (Wilcoxon sign-rank, $p<0.05$ ), $\mathrm{NaCl}$ (Wilcoxon sign-rank, $p<0.05$ ), or odorized water (Wilcoxon sign-rank, $p<0.05$ ) becomes significantly different from water alone after the first $500 \mathrm{~ms}$, and continued (except for a short $1-1.25 \mathrm{~s}$ interval) for the rest of the $2 \mathrm{~s}$ palatability processing period. These results suggest that the similarity between activity evoked by odorants and the activity evoked by palatable tastants was not exclusively explained by the presence of water in the odorized solutions.

To further investigate the relationship between activity evoked by odorized water and activity evoked by pairs of palatable or aversive tastants, we modified the palatability index formula (for details, see Materials and Methods), by substituting the odorants (isoamyl acetate and benzaldehyde) for either sucrose and $\mathrm{NaCl}$ or citric acid and quinine (Fig. $6 B$ ). This procedure allowed us to quantify the pairwise difference in activity evoked by odorants and tastants. Comparing odorants to aversive tastants led to a modified palatability index that was significantly above baseline (Fig. $6 \mathrm{~B}$, purple solid line, black bars, Wilcoxon rank-sum, $p<0.05$ ), indicating that the activity evoked by odorants was different from that evoked by aversive tastants. On the contrary, comparing odorants to palatable tastants revealed no significant difference (i.e., the modified palatability index was not significantly different from baseline-purple dotted line, Wilcoxon rank-sum, $p>0.05$ ), showing that odorants and palatable tastants evoked similar activity. The two modified palatability indices were significantly different (Wilcoxon sign-rank, $p<$ 0.01 ). Together, these results show that solutions of odorants in water can be processed similarly to pleasant tastants by a palatability-coding group of neurons in GC.

We next sought to behaviorally determine whether rats found the solutions of water and odorants palatable or aversive in our experimental conditions. We used a brief access task, which measures licking activity evoked by the brief presentation of different solutions and is considered a standard test to assess preference across multiple stimuli (Smith, 2001). Freely moving rats $(n=3)$, which had previous experience with all of the chemosensory stimuli, were trained to wait for pseudo-randomized presentations of the four tastants, the two odorants in water, and water. Each trial consisted of a $15 \mathrm{~s}$ window to lick the spout. The mean number of licks per $15 \mathrm{~s}$ presentation was used as a measure of stimulus preference (Sadacca et al., 2012). Rats licked significantly less to the aversive tastants of citric acid and quinine than to any of the other chemosensory stimuli (one-way ANOVA, $p<$ 0.01 ). Figure $6 C$ shows that rats exhibited significantly fewer licks to citric acid $(3.88 \pm 0.81, t$ test, $p<0.01)$ and quinine $(5.00 \pm$ $1.32, t$ test, $p<0.01)$ compared with water $(62.00 \pm 5.59)$. All the other solutions evoked either the same amount of licking $(\mathrm{NaCl}$ : $60.45 \pm 6.59, t$ test, $p>0.05$; isoamyl acetate: $64.12 \pm 4.48, t$ test, $p>0.05$; benzaldehyde: $65.56 \pm 5.00, t$ test, $p>0.05$ ) or a larger amount of licking (sucrose 70.68 $\pm 3.56, t$ test, $p<0.01$ ) than water. These behavioral findings confirmed the results from the neural activity of the bimodal neurons (i.e., odorants dissolved in water are preferred over aversive tastants).

\section{Discussion}

Our findings reveal how neurons in GC represent gustatory and olfactory signals originating from the mouth. Intraoral delivery of gustatory and olfactory stimuli unveiled three populations of chemoresponsive neurons in GC: unimodal gustatory (tasteonly), unimodal olfactory (odor-only), and a bimodal group that responded to both stimuli. Analyses of respiration and disruption of olfactory function with a nasal inactivation protocol con- firmed that GC responses to intraoral odorants were due to retronasal olfaction. Unimodal and bimodal taste-selective populations exhibited different taste coding properties, including taste selectivity, tuning, and palatability. Unlike unimodal tasteonly neurons, bimodal neurons encoded taste palatability. Bimodal neurons exhibited odor-evoked activity similar to that evoked by palatable tastants, suggesting that the odorants dissolved into water were palatable. This finding was behaviorally validated with a brief access task. These results provide novel evidence of how GC neurons encode the chemosensory components of flavor and identify a bimodal, palatability-coding population of neurons that may participate in linking odorants with taste qualities.

\section{A subset of neurons in GC encode intraoral odorants}

We observed that $15 \%$ of neurons in GC could respond to the intraoral delivery of odorants. This number should not be considered absolute, as a wider array of olfactory stimuli would likely have unveiled additional odor-responsive neurons. However, our choice of stimuli was limited by the number of odorants known to be tasteless (Slotnick et al., 1997; Gautam and Verhagen, 2012) to avoid experimental confounds.

Retronasal olfaction is a necessary component of flavor perception (Verhagen and Engelen, 2006; Prescott, 2012). In this study, we ensured that odorants were sampled retronasally, as all chemosensory stimuli were delivered directly into the mouth. Using a nasal inactivation technique, we confirmed that GC responses to intraoral odorants depended on olfaction. This manipulation significantly reduced the proportion of odor-responsive neurons without affecting the proportion of taste responses.

Retronasal olfaction is also dependent upon respiration (Masaoka et al., 2010; Gautam and Verhagen, 2012). The physical action required for retronasal olfaction predicted that odorevoked responses would occur after exhalation. In a subset of sessions, we recorded respiration and GC single-unit activity during the intraoral delivery of chemosensory stimuli. We found that both unimodal odor-only and bimodal neurons in GC exhibited odor-evoked activity significantly after the first exhalation after stimulus delivery. However, bimodal neurons also showed an initial significant response to intraoral stimulus delivery that occurred before the significant odor-evoked activity (Fig. 3B). Neurons in GC are known to respond to tactile stimulation of the mouth and tongue (Yamamoto et al., 1981; Wang and Ogawa, 2002), and previous experiments using uncued intraoral tastant deliveries showed that early taste-evoked activity $(\sim 0-200 \mathrm{~ms})$ in GC reflects a somatosensory component (Katz et al., 2001; Samuelsen et al., 2013). Therefore, the early sharp activity evoked by intraoral odorant delivery in bimodal neurons may, in part, be due to physical stimulation of the oral cavity. This early somatosensory component was not represented in the unimodal odoronly population. Together, our data show that odor-evoked activity in GC depends on olfaction and suggests that bimodal GC neurons, which are responsive to both retronasally sampled odorants and tastes, may play an important role in the perception of flavor.

\section{Chemosensory multimodality in GC}

Electrophysiological recordings have shown that neurons in GC not only encode gustatory stimuli but also process thermal (Kadohisa et al., 2005), visceral (Hanamori et al., 1998), visual (Ifuku et al., 2006; Vincis and Fontanini, 2016), somatosensory (Verhagen et al., 2005; Vincis and Fontanini, 2016), auditory signals (Samuelsen et al., 2012; Gardner and Fontanini, 2014; 
Kusumoto-Yoshida et al., 2015), and orthonasally delivered odors (Vincis and Fontanini, 2016). These multimodal responses are thought to result from anatomical connections between GC and other cortical, thalamic, and limbic areas (Allen et al., 1991; Shi and Cassell, 1998; Haley et al., 2016). Particularly relevant to our findings of intraoral odorant responses is the interconnectivity between GC and regions of the olfactory sensory pathway: the main olfactory bulb (Shipley and Geinisman, 1984) and piriform cortex (PIR) (Saper, 1982; Johnson et al., 2000). The fast latency of GC odor-evoked responses following exhalation in unimodal odor-only neurons suggests that responses to intraoral odorants may result from direct sensory input. Although electrical stimulation of the main olfactory bulb activates neurons in GC (Cinelli et al., 1987), little is understood about this bulbar projection. Another likely source of olfactory sensory input to GC is via PIR, an area with strong reciprocal connections to main olfactory bulb and GC (Haberly and Price, 1978). Recently, studies have found that PIR neurons encode gustatory signals (Maier et al., 2012) and optogenetic suppression of GC modulates PIR odor-evoked responses while disrupting olfactory-dependent behavior (Maier et al., 2015). These findings suggest that sensory encoding of the components of flavor may result from a coordinated relationship between primary chemosensory cortical areas.

Higher-order cortical, thalamic, or limbic areas may also contribute to GC multimodal responses to odorants. The basolateral amygdala, OFC, and mediodorsal thalamus have been proposed to be involved in integrating gustatory and olfactory signals to form the percept of flavor (Small, 2012). The basolateral amygdala, reciprocally connected to both GC and PIR (Allen et al., 1991; Johnson et al., 2000), processes the psychological aspects of taste (Piette et al., 2012; Samuelsen et al., 2012) and has been proposed as the likely driver of gustatory activity in PIR (Maier et al., 2012). Human neuroimaging (De Araujo et al., 2003; Small et al., 2004) and single-unit recordings in nonhuman primates (Rolls and Baylis, 1994) have shown gustatory and olfactory interactions in OFC. The mediodorsal thalamus receives olfactory information from PIR (Plailly et al., 2008), is reciprocally connected to GC, basolateral amygdale, and OFC (Krettek and Price, 1977; Ray and Price, 1992), and humans with mediodorsal thalamus lesions report lower hedonic ratings for experienced odorants and flavors (Rousseaux et al., 1996; Asai et al., 2008; Sela et al., 2009; Tham et al., 2011). The interaction between these higher-order areas and primary sensory cortices could modulate the response to odorants in GC, particularly in the case of palatability coding, bimodal neurons identified in this study.

\section{Modality-dependent differences in taste processing}

A comparison of taste coding between taste-only and bimodal neurons revealed several differences. In both groups, the majority of neurons encoded more than one taste quality. However, significantly more unimodal taste-only neurons $(\sim 25 \%)$ encoded a single taste quality compared with bimodal neurons $(\sim 9 \%)$. Analysis of the time course of tuning found that, $1 \mathrm{~s}$ after taste delivery, more unimodal neurons encoded single tastants than multiple tastants, a behavior consistent with previous reports (Samuelsen et al., 2013). This progressive narrowing of tuning poststimulus delivery was not observed for bimodal neurons. An even more striking difference was observed with palatability coding, where bimodal neurons encoded palatability significantly better than unimodal neurons. Bimodal neurons were not the only ones capable of encoding palatability; however, a significantly larger proportion of bimodal neurons coded for palatabil- ity relative to taste-only neurons. Bimodal neurons also showed a bias toward palatable tastants, with prolonged responses to sucrose and $\mathrm{NaCl}$ and comparatively shorter responses to citric acid and quinine. Odorants dissolved in water evoked patterns of activity more similar to palatable tastants than aversive tastants in the population of bimodal neurons. This result led to the prediction that rats would perceive the water solutions of isoamyl acetate and benzaldehyde as palatable or at least not aversive, in our experimental conditions. Indeed, this prediction was confirmed by a brief access task that found rats lick for these odorants as much as they lick for water or palatable tastants, and significantly more than they lick for aversive tastants. Our results suggest that the two groups of taste-selective neurons participate in different facets of taste processing. Where unimodal taste-only neurons may primarily encode taste quality, bimodal neurons may engage in coding palatability, playing a role in the "flavor network" (Small and Green, 2012) by linking odorants to taste qualities.

Why is odorized water palatable? A parsimonious explanation might attribute palatability exclusively to the presence of water. The palatability of water to thirsty rats may be enough to make odorized water palatable and drive its consumption. This consideration implies that the odors themselves, isoamyl acetate and benzaldehyde, are not inherently aversive. It is also possible that the palatability-related activity observed for isoamyl acetate and benzaldehyde may be the result of experience and derive from pairing the odors with water for multiple days (Gautam and Verhagen, 2010). According to this model, water-odor pairings in thirsty rats could lead odors to acquire the palatability of water. In this regard, odors could become palatable themselves and synergize with water in promoting consumption. Our data showing that odorants dissolved in water evoke activity similar to sucrose and $\mathrm{NaCl}$, but different from water alone, is consistent with this possibility. However, these results do not rule out the importance of water in driving palatability. Of course, more experiments will be necessary to ultimately address this important point.

Whether responses to odorants and tastant are hard wired or the result of learning remains an open question. Given the evidence of cross-modal plasticity in GC (Gardner and Fontanini, 2014; Vincis and Fontanini, 2016), it is highly likely that pairing odorants with tastants may increase the pool of neurons responding to both modalities. Hence, learning is likely to play a central role in shaping taste-odor convergence, as well as palatability coding.

To investigate the basic chemosensory properties of GC neurons, we intentionally presented tastants and odorants separately; we did not present taste-odor mixtures. Human neuroimaging and psychophysical experiments have found both superadditive (Small et al., 2004; Burseg et al., 2010) and mixture suppression effects (Stevenson et al., 1999) to taste-odor mixtures. Although we identified taste processing differences in unimodal and bimodal neurons in GC, it remains to be determined how chemoresponsive neurons in GC represent taste-odor mixtures.

In conclusion, our findings demonstrate that neurons in GC integrate gustatory and olfactory sensory signals from the mouth and identify different neuronal subgroups that may perform distinct functions in the flavor network. These findings represent the foundation for a variety of new research directions aimed at investigating multisensory processing, particularly exploring the role of GC multimodality in processing flavor. 


\section{References}

Aimé P, Duchamp-Viret P, Chaput MA, Savigner A, Mahfouz M, Julliard AK (2007) Fasting increases and satiation decreases olfactory detection for a neutral odor in rats. Behav Brain Res 179:258-264. CrossRef Medline

Allen GV, Saper CB, Hurley KM, Cechetto DF (1991) Organization of visceral and limbic connections in the insular cortex of the rat. J Comp Neurol 311:1-16. CrossRef Medline

Asai H, Udaka F, Hirano M, Ueno S (2008) Odor abnormalities caused by bilateral thalamic infarction. Clin Neurol Neurosurg 110:500-501. CrossRef Medline

Burseg KM, Camacho S, Knoop J, Bult JH (2010) Sweet taste intensity is enhanced by temporal fluctuation of aroma and taste, and depends on phase shift. Physiol Behav 101:726-730. CrossRef Medline

Cazakoff BN, Lau BY, Crump KL, Demmer HS, Shea SD (2014) Broadly tuned and respiration-independent inhibition in the olfactory bulb of awake mice. Nat Neurosci 17:569-576. CrossRef Medline

Cerf-Ducastel B, Murphy C (2001) fMRI activation in response to odorants orally delivered in aqueous solutions. Chem Senses 26:625-637. CrossRef Medline

Cerf-Ducastel B, Van de Moortele PF, MacLeod P, Le Bihan D, Faurion A (2001) Interaction of gustatory and lingual somatosensory perceptions at the cortical level in the human: a functional magnetic resonance imaging study. Chem Senses 26:371-383. CrossRef Medline

Cinelli AR, Ferreyra-Moyano H, Barragan E (1987) Reciprocal functional connections of the olfactory bulbs and other olfactory related areas with the prefrontal cortex. Brain Res Bull 19:651-661. CrossRef Medline

Cohen JY, Haesler S, Vong L, Lowell BB, Uchida N (2012) Neuron-typespecific signals for reward and punishment in the ventral tegmental area. Nature 482:85-88. CrossRef Medline

de Araujo IE, Rolls ET, Kringelbach ML, McGlone F, Phillips N (2003) Taste-olfactory convergence, and the representation of the pleasantness of flavour, in the human brain. Eur J Neurosci 18:2059-2068. CrossRef Medline

Fontanini A, Grossman SE, Figueroa JA, Katz DB (2009) Distinct subtypes of basolateral amygdala taste neurons reflect palatability and reward. J Neurosci 29:2486-2495. CrossRef Medline

Fortis-Santiago Y, Rodwin BA, Neseliler S, Piette CE, Katz DB (2010) Statedependence of olfactory perception as a function of taste cortical inactivation. Nat Neurosci 13:158-159. CrossRef Medline

Friedrich RW, Korsching SI (1997) Combinatorial and chemotopic odorant coding in the zebrafish olfactory bulb visualized by optical imaging. Neuron 18:737-752. CrossRef Medline

Gardner MP, Fontanini A (2014) Encoding and tracking of outcomespecific expectancy in the gustatory cortex of alert rats. J Neurosci 34: 13000-13017. CrossRef Medline

Gautam SH, Verhagen JV (2012) Direct behavioral evidence for retronasal olfaction in rats. PLoS One 7:e44781. CrossRef Medline

Gautam SH, Verhagen JV (2010) Evidence that the sweetness of odors depends on experience in rats. Chem Senses 35:767-776. CrossRef Medline

Haberly LB, Price JL (1978) Association and commissural fiber systems of the olfactory cortex of the rat. J Comp Neurol 178:711-740. CrossRef Medline

Haley MS, Fontanini A, Maffei A (2016) Laminar- and target-specific amygdalar inputs in rat primary gustatory cortex. J Neurosci 36:26232637. CrossRef Medline

Hanamori T, Kunitake T, Kato K, Kannan H (1998) Responses of neurons in the insular cortex to gustatory, visceral, and nociceptive stimuli in rats. J Neurophysiol 79:2535-2545. Medline

Ifuku H, Nakamura T, Hirata S, Ogawa H (2006) Neuronal activities in the reward phase in primary and higher-order gustatory cortices of monkeys. Neurosci Res 55:54-64. CrossRef Medline

Jezzini A, Mazzucato L, La Camera G, Fontanini A (2013) Processing of hedonic and chemosensory features of taste in medial prefrontal and insular networks. J Neurosci 33:18966-18978. CrossRef Medline

Johnson DM, Illig KR, Behan M, Haberly LB (2000) New features of connectivity in piriform cortex visualized by intracellular injection of pyramidal cells suggest that "primary" olfactory cortex functions like "association" cortex in other sensory systems. J Neurosci 20:6974-6982. Medline

Julliard AK, Chaput MA, Apelbaum A, Aimé P, Mahfouz M, Duchamp-Viret $P$ (2007) Changes in rat olfactory detection performance induced by orexin and leptin mimicking fasting and satiation. Behav Brain Res 183: 123-129. CrossRef Medline

Kadohisa M, Rolls ET, Verhagen JV (2005) Neuronal representations of stimuli in the mouth: the primate insular taste cortex, orbitofrontal cortex and amygdala. Chem Senses 30:401-419. CrossRef Medline

Katz DB, Simon SA, Nicolelis MA (2001) Dynamic and multimodal responses of gustatory cortical neurons in awake rats. J Neurosci 21:44784489. Medline

Kobayakawa T, Ogawa H, Kaneda H, Ayabe-Kanamura S, Endo H, Saito S (1999) Spatio-temporal analysis of cortical activity evoked by gustatory stimulation in humans. Chem Senses 24:201-209. CrossRef Medline

Krettek JE, Price JL (1977) The cortical projections of the mediodorsal nucleus and adjacent thalamic nuclei in the rat. J Comp Neurol 171:157-191. CrossRef Medline

Kusumoto-Yoshida I, Liu H, Chen BT, Fontanini A, Bonci A (2015) Central role for the insular cortex in mediating conditioned responses to anticipatory cues. Proc Natl Acad Sci U S A 112:1190-1195. CrossRef Medline

Li JX, Yoshida T, Monk KJ, Katz DB (2013) Lateral hypothalamus contains two types of palatability-related taste responses with distinct dynamics. J Neurosci 33:9462-9473. CrossRef Medline

Liu H, Fontanini A (2015) State dependency of chemosensory coding in the gustatory thalamus (VPMpc) of alert rats. J Neurosci 35:15479-15491. CrossRef Medline

Maier JX, Wachowiak M, Katz DB (2012) Chemosensory convergence on primary olfactory cortex. J Neurosci 32:17037-17047. CrossRef Medline

Maier JX, Blankenship ML, Li JX, Katz DB (2015) A multisensory network for olfactory processing. Curr Biol 25:2642-2650. CrossRef Medline

Mak YE, Simmons KB, Gitelman DR, Small DM (2005) Taste and olfactory intensity perception changes following left insular stroke. Behav Neurosci 119:1693-1700. CrossRef Medline

Masaoka Y, Satoh H, Akai L, Homma I (2010) Expiration: the moment we experience retronasal olfaction in flavor. Neurosci Lett 473:92-96. CrossRef Medline

O’Doherty JP, Rolls ET, Francis S, Bowtell R, McGlone F (2001) Representation of pleasant and aversive taste in the human brain. J Neurophysiol 85:1315-1321. Medline

Piette CE, Baez-Santiago MA, Reid EE, Katz DB, Moran A (2012) Inactivation of basolateral amygdala specifically eliminates palatability-related information in cortical sensory responses. J Neurosci 32:9981-9991. CrossRef Medline

Plailly J, Howard JD, Gitelman DR, Gottfried JA (2008) Attention to odor modulates thalamocortical connectivity in the human brain. J Neurosci 28:5257-5267. CrossRef Medline

Poellinger A, Thomas R, Lio P, Lee A, Makris N, Rosen BR, Kwong KK (2001) Activation and habituation in olfaction: an fMRI study. Neuroimage 13:547-560. CrossRef Medline

Prescott J (2012) Chemosensory learning and flavour: perception, preference and intake. Physiol Behav 107:553-559. CrossRef Medline

Ray JP, Price JL (1992) The organization of the thalamocortical connections of the mediodorsal thalamic nucleus in the rat, related to the ventral forebrain-prefrontal cortex topography. J Comp Neurol 323:167-197. CrossRef Medline

Rebello MR, Kandukuru P, Verhagen JV (2015) Direct behavioral and neurophysiological evidence for retronasal olfaction in mice. PLoS One 10: 16-19. CrossRef Medline

Rolls ET (2005) Taste, olfactory, and food texture processing in the brain, and the control of food intake. Physiol Behav 85:45-56. CrossRef Medline

Rolls ET, Baylis LL (1994) Gustatory, olfactory, and visual convergence within the primate orbitofrontal cortex. J Neurosci 14:5437-5452. Medline

Rousseaux M, Muller P, Gahide I, Mottin Y, Romon M (1996) Disorders of smell, taste, and food intake in a patient with a dorsomedial thalamic infarct. Stroke 27:2328-2330. CrossRef Medline

Rozin P (1982) "Taste-smell confusions" and the duality of the olfactory sense. Percept Psychophys 31:397-401. CrossRef Medline

Sadacca BF, Rothwax JT, Katz DB (2012) Sodium concentration coding gives way to evaluative coding in cortex and amygdala. J Neurosci 32: 9999-10011. CrossRef Medline

Samuelsen CL, Gardner MP, Fontanini A (2012) Effects of cue-triggered expectation on cortical processing of taste. Neuron 74:410-422. CrossRef Medline

Samuelsen CL, Gardner MP, Fontanini A (2013) Thalamic contribution to 
cortical processing of taste and expectation. J Neurosci 33:1815-1827. CrossRef Medline

Saper CB (1982) Convergence of autonomic and limbic connections in the insular cortex of the rat. J Comp Neurol 210:163-173. CrossRef Medline

Savic I, Gulyas B, Larsson M, Roland P (2000) Olfactory functions are mediated by parallel and hierarchical processing. Neuron 26:735-745. CrossRef Medline

Sela L, Sacher Y, Serfaty C, Yeshurun Y, Soroker N, Sobel N (2009) Spared and impaired olfactory abilities after thalamic lesions. J Neurosci 29: 12059-12069. CrossRef Medline

Shi C, Cassell MD (1998) Cortical, thalamic, and amygdaloid connections of the anterior and posterior insular cortices. J Comp Neurol 399: 440-468. Medline

Shipley MT, Geinisman Y (1984) Anatomical evidence for convergence of olfactory, gustatory, and visceral afferent pathways in mouse cerebral cortex. Brain Res Bull 12:221-226. CrossRef Medline

Slotnick B, Westbrook F, Darling F (1997) What the rat's nose tells the rat's mouth: long delay aversion conditioning with aqueous odors and potentiation of taste by odors. Anim Learn Behav 25:357-369. CrossRef

Small DM (2012) Flavor is in the brain. Physiol Behav 107:540-552. CrossRef Medline

Small DM, Green BG (2012) A proposed model of a flavor modality (Murray $\mathrm{MM}$, Wallace $\mathrm{MT}$, eds). Boca Raton, FL: CRC.

Small DM, Zald DH, Jones-Gotman M, Zatorre RJ, Pardo JV, Frey S, Petrides M (1999) Human cortical gustatory areas: a review of functional neuroimaging data. Neuroreport 10:7-14. CrossRef Medline

Small DM, Gregory MD, Mak YE, Gitelman D, Mesulam MM, Parrish T (2003) Dissociation of neural representation of intensity and affective valuation in human gustation. Neuron 39:701-711. CrossRef Medline

Small DM, Voss J, Mak YE, Simmons KB, Parrish T, Gitelman D (2004) Experience-dependent neural integration of taste and smell in the human brain. J Neurophysiol 92:1892-1903. CrossRef Medline

Small DM, Veldhuizen MG, Felsted J, Mak YE, McGlone F (2008) Separable substrates for anticipatory and consummatory food chemosensation. Neuron 57:786-797. CrossRef Medline

Smith JC (2001) The history of the "Davis Rig." Appetite 36:93-98. CrossRef
Smith JC, Davis JD, O’Keefe GB (1992) Lack of an order effect in brief contact taste tests with closely spaced test trials. Physiol Behav 52:11071111. CrossRef Medline

Stevenson RJ, Prescott J, Boakes RA (1999) Confusing tastes and smells: how odours can influence the perception of sweet and sour tastes. Chem Senses 24:627-635. CrossRef Medline

Tham WW, Stevenson RJ, Miller LA (2011) The impact of mediodorsal thalamic lesions on olfactory attention and flavor perception. Brain Cogn 77:71-79. CrossRef Medline

Tong J, Mannea E, Aimé P, Pfluger PT, Yi CX, Castaneda TR, Davis HW, Ren X, Pixley S, Benoit S, Julliard K, Woods SC, Horvath TL, Sleeman MM, D’Alessio D, Obici S, Frank R, Tschöp MH (2011) Ghrelin enhances olfactory sensitivity and exploratory sniffing in rodents and humans. J Neurosci 31:5841-5846. CrossRef Medline

Veldhuizen MG, Nachtigal D, Teulings L, Gitelman DR, Small DM (2010) The insular taste cortex contributes to odor quality coding. Front Hum Neurosci 4:1-11. CrossRef Medline

Verhagen JV, Engelen L (2006) The nerocognitive bases of human food perception: sensory integration. Neurosci Biobehav Rev 30:613-650. CrossRef Medline

Verhagen JV, Giza BK, Scott TR (2005) Effect of amiloride on gustatory responses in the ventroposteromedial nucleus of the thalamus in rats. J Neurophysiol 93:157-166. CrossRef Medline

Vincis R, Fontanini A (2016) Associative learning changes cross-modal representations in the gustatory cortex. Elife 5:e16420. CrossRef Medline

Wachowiak M, Cohen LB (2001) Representation of odorants by receptor neuron input to the mouse olfactory bulb. Neuron 32:723-735. CrossRef Medline

Wang X, Ogawa H (2002) Columnar organization of mechanoreceptive neurons in the cortical taste area in the rat. Exp Brain Res 147:114-123. CrossRef Medline

Wesson DW (2013) Sniffing behavior communicates social hierarchy. Curr Biol 23:575-580. CrossRef Medline

Yamamoto T, Yuyama N, Kawamura Y (1981) Cortical neurons responding to tactile, thermal and taste stimulations of the rat's tongue. Brain Res 221:202-206. CrossRef Medline 